\title{
Bounds on Exponentials of Local Number Operators in Quantum Statistical Mechanics
}

\author{
Yong Moon Park * \\ Department of Mathematics, Yonsei University, Seoul, Korea
}

\begin{abstract}
We consider the quantum systems of interacting Bose particles confined to a bounded region $\Lambda$ of the configuration spaces $\mathbb{R}^{v}$. For a class of superstable interactions we obtain bounds on exponentials of local number operators for any temperature and activity. The method we use is the Wiener integral formalism in statistical mechanics. As a consequence any thermodynamic limit states are entire analytic and locally normal in the CCR algebra. In some cases these are modular states.
\end{abstract}

\section{Introduction}

In this paper we study a class of superstable interactions in quantum statistical mechanics with Bose-Einstein statistics. There have been extensive studies on the thermodynamic limit in statistical mechanics of interacting quantum systems and fairly satisfactory results have been obtained for the thermodynamic functions $[2,12]$. The results concerning the equilibrium states for such systems are less satisfactory. In the dilute regime, detailed properties of the thermodynamic limit states have been obtained for various classes of interactions $[2-4,6,15]$. There are also some results on the thermodynamic limits of the finite volume Gibbs states of interacting Bose particles for the charge conjugation invariant systems [5] and for the repulsive systems (with activity less than one) [1]. For the classical systems with superstable interactions, Ruelle established uniform bounds of the finite volume correlation functions [13]. Using the bounds he obtained various results on the infinite volume equilibrium states and the pressure. The results have been extended to unbounded classical spin systems $[14,8]$. The main purpose of this paper is to extend Ruelle's results to quantum statistical mechanics for interacting Bose particles. The method we develop can be extended easily to unbounded quantum spin systems and will appear elsewhere [10].

We give a brief discussion of the main result. Let $\rho_{A}$ be the Gibbs equilibrium states for a system of interacting Bose particles confined to a bounded region $\Lambda$

^ Research supported in part by a grant from Korean Science Foundation 
of the configuration space $\mathbb{R}^{v}$ and let $N_{B}$ be the local number operators for $B \subset \Lambda$. We shall assume that the interaction between particles is given by a pair potential $\Phi$. Under the assumption that $\Phi$ satisfies one of the following conditions:

(1) $\Phi$ is a positive function with non-zero around the origin.

(2) There are constants $\gamma_{1}, \gamma_{2}$ and $\lambda$ such that

$$
\begin{aligned}
& \Phi(x) \geqq|x|^{-\lambda}, \quad|x| \leqq \gamma_{1}, \\
& \Phi(x)>-|x|^{-(v+2)}, \quad|x| \geqq \gamma_{2},
\end{aligned}
$$

where $\lambda>0$ will depend on $v$ (e.g. $\lambda>6$ for $v=3$ ), we then obtain the bound of the form $(v<4$ in case $(2))$

$$
\rho_{\Lambda}\left(e^{|\alpha| N_{B}}\right) \leqq \exp [A(B, \alpha)],
$$

where $A(B, \alpha)$ depend only on the diameter of $B$ and $|\alpha|$. We will give the exact definition of models, conditions on the interactions and the main result in Sect. II. As a consequence of the bounds it will follow that any limit point $\rho$ of the states $\rho_{\Lambda}$ in the weak ${ }^{\star}$ topology is entire analytic and locally normal state on the CCR algebra.

The basic ingredients of the method we use are the representation of the partition function in terms of the Wiener integrals $[2,6,7]$, and a modification of Ruelle's method used for classical systems [13]. In comparison to the classical systems, the quantum systems are much more difficult to handle. One has to solve problems originated from

a) quantum statistics,

b) fluctuations of Wiener paths.

In order to control the above, we will use the fact that the Wiener measure of a subset of the paths with large fluctuations is small. On the other hand, the system behaves like classical on a subset of paths with small fluctuations. The basic idea is a decomposition of the Wiener space $\Omega^{n}$ of $n$-paths into disjoint subsets. On each subset we utilize the above observation to obtain uniform bounds. For the details, see Sect. IV. 1-Sect. IV.4.

We wish to make a few remarks on merits and demerits of our method.

Remark. (a) Using our method (with some necessary modifications) one may be able to get uniform bounds of other objects such as local perturbations of Hamiltonians. These kinds of bounds are expected to be useful to investigate more detailed properties of the systems.

(b) We were unable to obtain pointwise bounds on reduced density matrices. By establishing decay properties one ought to be able to see whether the systems do exhibit Bose-Einstein condensation. We believe that the method we use can be extended to give these bounds.

(c) Because of technical reasons, we need additional assumptions in (2) ((2.2.2) and (2.2.3) in Assumption A in Sect. II.2) more than superstability. These conditions on the interactions seem to be optimal in our method. We would like to know whether the additional assumptions (and the restriction $v<4$ ) are necessary.

(d) The Wiener integral formalism we use seems to be inappropriate to handle 
the systems of interacting Fermi particles. These systems involve some negative terms in the Wiener integral formalism $[2,5,6]$. Thus it would be nice to develop a method in the pure operator approach, i.e., in the second quantization formalism. The method should also given an answer to the problem in Remark (c).

We list the contents of the paper. In Sect. II.1, we introduce notations and the definition of models. We then list the assumptions on the interactions (Assumption $A$ and Assumption $A^{\prime}$ ) in Sect. II.2. We also give some discussions on the assumptions in the space dimensions $v$. In Sect. II.3, we give the main result (Theorem II.3.1), and then we derive consequences from the main result such as entire analyticity, locally normality and modularity of the thermodynamic limit states (Theorem II.3.3 and Theorem II.3.4).

In Sect. III.1 we introduce the Wiener integral formalism in quantum statistical mechanics. Some estimates on the Wiener measure are given in Sect. III.2. In Sect. III.3 we introduce ideas of partial symmetry spaces which will be needed to control quantum statistics later.

Section IV is devoted to the proof of the main result. The expansion method used in this paper is developed in Sect. IV.1. We decomposed the space of $n$-Wiener paths (trajectories) into disjoint subsets to expand the partition function. In Sect. IV.2. We give the basic ideas how to obtain uniform bounds on each subset. With an assumption of one estimate (Theorem IV.2.1) we prove the main theorem in Sect. IV.3. Section IV.4 is devoted to establishing the basic estimate, Theorem IV.2.1, in this paper. The additional assumptions more than superstability on the interactions are needed to get the basic estimate.

Finally in Sect. V we give some discussion on the reason why conditions (2.2.2), (2.2.3) and the restriction $v<4$ for the potentials satisfying (2) seem to be optimal.

\section{The Definition of Models and the Main Results}

\section{II.1. Some Notations and Definitions}

We first introduce the Hilbert space, the Hamiltonian and the Gibbs equilibrium states for systems of interacting particles, which satisfy Bose-Einstein statistics, confined to a bounded open region $\Lambda$ of the configuration space $\mathbb{R}^{v}$. Let

$$
\mathscr{H}_{n}^{(s)}(\Lambda)=\bigotimes_{i=1}^{n} L^{2}\left(\Lambda, d^{v} x_{i}\right)
$$

be the subspace of $L^{2}\left(\Lambda^{n}, d^{n v} x\right)$ formed by the totally symmetric functions of $n$-variables $x_{i} \in \Lambda$. The associated Fock space

$$
\mathscr{F}^{(s)}(\Lambda)=\underset{n \geqq 0}{\oplus} \mathscr{H}_{n}^{(s)}(\Lambda)
$$

describes the states of an arbitrary number of particles. The total Hamiltonian is given by

$$
H_{\Lambda}=\underset{n \geqq 0}{\oplus} H_{\Lambda}^{(n)}
$$


in terms of the $n$-particle Hamiltonian $H_{\Lambda}^{(n)}$, which has the form

$$
H_{\Lambda}^{(n)}=-\frac{1}{2} \sum_{i=1}^{n} \Delta_{\Lambda, i}+U\left(\left(x_{n}\right)\right)
$$

where $\Delta_{\Lambda, i}$ is the Laplacian in the variable $x_{i}$ with 0 -Dirichlet boundary condition on the boundary $\partial \Lambda$ of $\Lambda$, and the interaction energy of $n$ particles at the point $(x)_{n}=\left(x_{1}, x_{2}, \ldots, x_{n}\right)$ is given by

$$
U\left((x)_{n}\right)=\sum_{1 \leqq i<j \leqq n} \Phi\left(x_{i}-x_{j}\right),
$$

where $\Phi$ is a two body potential between particles. If the interaction operator satisfies the stability condition

$$
U\left((x)_{n}\right) \geqq-B n,
$$

for all $n$ and $(x)_{n} \in \mathbb{R}^{n v}, H_{\Lambda}^{(n)}$ is a self-adjoint operator on $\mathscr{H}_{n}^{(s)}(\Lambda)$. Self-adjointness of $H_{A}^{(n)}$ ensures that the total Hamiltonian $H_{\Lambda}$ is a self-adjoint operator on $\mathscr{F}^{(s)}(\Lambda)$ $[2,12]$.

Let $B \subset \Lambda$ be a bounded region in $\mathbb{R}^{v}$. We define the local number operators $N_{B}$ by

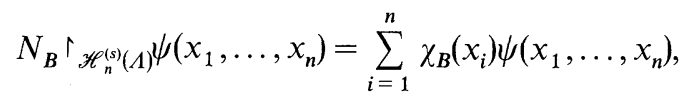

where $\chi_{B}$ is the characteristic function of $B$. We note that for any $\psi \in \mathscr{H}_{n}^{(s)}(\Lambda)$, $N_{A} \psi=n \psi$. If the interaction is superstable in the sense that, if $\Lambda$ is a cube of sufficiently large volume and $\left(x_{n}\right) \in \Lambda$, then

$$
U\left((x)_{n}\right) \geqq A \frac{n^{2}}{|\Lambda|}-B n
$$

with $A, B>0$, then the partition function defined by

$$
\Xi_{\Lambda}=\operatorname{Tr}_{\mathscr{F}(s)}\left(\exp \left[-\beta\left(H_{\Lambda}-\mu N_{\Lambda}\right)\right)\right.
$$

is bounded by $1 \leqq \Xi_{\Lambda} \leqq \exp [a|\Lambda|]$ for all $\beta \in(0, \infty)$ and $\mu \in \mathbb{R}[6,12]$.

Therefore if the interaction is superstable, one may introduce the finite-volume Gibbs states $\rho_{\Lambda}$ by

$$
\rho_{\Lambda}(A)=\Xi_{\Lambda}^{-1} \operatorname{Tr}_{\mathscr{F}^{(s)}(\Lambda)}\left(A \exp \left[-\beta\left(H_{\Lambda}-\mu N_{\Lambda}\right)\right)\right.
$$

for any bounded operator $A$ on $\mathscr{F}^{(s)}(\Lambda)$, and for any $\beta \in(0, \infty), \mu \in \mathbb{R}$.

\section{II.2. The Assumptions on the Interactions}

We now list the assumptions on the interactions. We shall assume that the interaction between particles is given by a pair potential $\Phi$ :

$$
U\left(x_{1}, \ldots, x_{n}\right)=\sum_{i<j}^{n} \Phi\left(x_{i}-x_{j}\right),
$$


where $\Phi$ is a Lebesgue measurable function which satisfies $\Phi(x)=\Phi(-x)$ and which may take real values and the value $+\infty$.

Let $0<\lambda \in \mathbb{R}$. For every $r \in Z^{v}$ we define a cube

$$
Q(r)=\left\{x \in \mathbb{R}^{v}:\left(r^{i}-\frac{1}{2}\right) \lambda \leqq x^{i}<\left(r^{i}+\frac{1}{2}\right) \lambda\right\} .
$$

These cubes form a partition of $\mathbb{R}^{v}$. If $X \in\left(\mathbb{R}^{v}\right)^{m}$, we let $n(X, r)$ be the number of points of the sequence $X=\left(x_{1}, \ldots, x_{m}\right)$ which belong to $Q(r)$.

Assumption A. (a) Strong Superstability: There exist $A>0, B>0$ such that if $\mathscr{R}$ is a finite subset of $Z^{v}$ and

$$
x_{1}, \ldots, x_{m} \in \bigcup_{r \in \mathscr{R}} Q(r), \quad X=\left(x_{1}, \ldots, x_{m}\right),
$$

then

$$
U(X) \geqq \sum_{r \in \mathscr{R}}\left[A n(X, r)^{p^{\prime}}-B n(X, r)\right]
$$

for $p^{\prime}(\geqq 2)$, which satisfies the inequality

$$
\frac{p^{\prime}}{p^{\prime}-1}<1+\frac{2\left(p^{\prime}-1\right)}{v p^{\prime}+(2-v)}
$$

(b) Strong lower regularity: There is a positive decreasing function $\varphi$ on $(0,+\infty)$ such that

$$
\int_{0}^{\infty} t^{v+1} \varphi(t) d t<\infty
$$

and for all $x \in \mathbb{R}^{v}$,

$$
\Phi(x) \geqq-\varphi(|x|)
$$

Assumption $A^{\prime}$. Positivity: $\Phi$ is a positive measurable function with the property that there exist constants $c>0$ and $d>0$ such that $\Phi(x)>c$ for $|x| \leqq d$.

We give a brief discussion on the assumptions on $\Phi$. Let $\Phi$ be of the form

$$
\Phi=\Phi_{1}+\Phi_{2},
$$

where $\Phi_{1}$ is a stable pair potential and $\Phi_{2}$ is a positive function. If $\Phi_{2}$ is a continuous function with $\Phi_{2}(0)>0$, then the strong superstability condition (2.2.2) holds with $p^{\prime}=2$. Moreover, if there exists $d>0$ such that

$$
\Phi_{2}(x) \geqq|x|^{-\lambda}, \quad \text { if }|x| \leqq d,
$$

then (2.2.2) holds with

$$
p^{\prime}=1+\lambda / v
$$

We refer to the references $[12,13]$ for more detailed discussions on the super-stable interactions. The inequality (2.2.3) came from the technical restrictions of our method in the proof of the main result. Roughly speaking we need non-zero $\lambda>0$ 
in (2.2.6) if $\Phi_{1} \neq 0$ in (2.2.5). An direct calculation shows that (2.2.3) implies

$$
\begin{aligned}
p^{\prime}>\frac{5+\sqrt{17}}{4}, & v=1, \\
p^{\prime}>\frac{3+\sqrt{5}}{2}, & v=2, \\
p^{\prime}>3, & v=3 .
\end{aligned}
$$

Notice that the right-hand side of (2.2.3) decreases as $v$ increasing. Thus we need stronger superstability conditions for higher dimensional spaces. On the other hand (2.2.4) implies that the attractive part of the potential should decay faster than $|x|^{-v-2}$. Thus the Lennard Jones type potentials

$$
\Phi(x)=a|x|^{-\alpha}-b|x|^{-\beta}, \quad a, b>0
$$

satisfy Assumption A under appropriate restrictions on $\alpha$ and $\beta$. For instance, $\alpha>6$ and $\alpha>\beta>5$ for $\nu=3$. For pure repulsive potentials satisfying Assumption $A^{\prime}$ one does not need any other restrictions. We will come back to the reason why we need (2.2.3) in Sect. IV.4 and Sect. V.

\section{II.3. The Main Result}

We first give our main theorem:

Theorem II.3.1. (a) Let the interaction satisfy Assumption A in Sect. II.2. For given $\beta \in(0, \infty)$ and $\mu \in \mathbb{R}$, let $\rho_{A}$ be the finite volume Gibbs state defined in (2.1.8) for the interaction. Then for any $B \subset \Lambda$ and $\alpha \in \mathbb{R}$, there is a constant $A(B,|\alpha|)$ such that, if $v<4$,

$$
\rho_{\Lambda}\left(e^{|\alpha| N_{B}}\right) \leqq e^{A(B,|\alpha|)}
$$

holds, where $A(B,|\alpha|)$ depends only on $\operatorname{diam}(B)$ and $|\alpha|$.

(b) If the interaction satisfies Assumption $A^{\prime}$, the same bound as the above holds for any $v \in \mathbb{N}$.

We will produce the proof of the above theorem in Sect. IV. Using the method (with some modifications if necessary) of this paper, one may be able to get the uniform bounds of other objects such as local perturbations of the interactions. These kinds of bounds may be useful to analyze the properties of the models in more detail. Because the proof of Theorem II.3.1. already involves considerable complications we do not establish other bounds here.

We next consider some consequences of Theorem II.3.1. We first establish the following result:

Proposition II.3.2. Let $B_{i}, i=1,2, \ldots, k$, be subsets of $\Lambda, B_{i} \subset \Lambda$. There is a constant $c$ such that

$$
\rho_{\Lambda}\left(\prod_{i=1}^{k} N_{B_{\imath}}\right) \leqq c k ! \prod_{i=1}^{k} N^{+}\left(B_{i}\right)
$$


where $\mathrm{N}^{+}(B)$ is the minimal number of the unit cubes which cover $B$.

Proof. Let $\left\{\Delta_{i, j}\right\}$ be the set of the disjoint half open unit cubes which covers $B_{i}$. Then

Thus

$$
N_{B_{\imath}}=\sum_{j} N_{B_{i} \cap \Delta_{i, j}} \leqq \sum_{\Delta_{\imath, J} \cap B_{i} \neq \phi} N_{\Delta_{i, j}} .
$$

$$
\rho_{\Lambda}\left(\prod_{i=1}^{k} N_{B_{i}}\right) \leqq \sum_{\Delta_{1, l_{1}} \cap \boldsymbol{B}_{1} \neq \phi} \ldots \sum_{\Delta_{k, l_{k}} \cap \boldsymbol{B}_{k} \neq \phi} \rho_{\Lambda}\left(\prod_{j=1}^{k} N_{\Delta_{i, i_{j}}}\right) .
$$

Using the abstract Hölder's inequality for trace norms [11], commutativity of local number operators and the cyclicity of the trace, we get

$$
\rho_{\Lambda}\left(\prod_{i=1}^{k} N_{\Delta_{i}}\right) \leqq \prod_{i=1}^{k}\left[\rho_{\Lambda}\left(N_{\Delta_{i}}^{k}\right)\right]^{1 / k}
$$

We now use the spectral theorem to obtain $\left\|N_{\Delta}^{k} \exp \left(-N_{\Delta}\right)\right\| \leqq k !$ (or one may use the Cauchy integral formula for $\rho_{\Lambda}\left(e^{z N_{\Delta}}\right)$. Thus it follows that

$$
\rho_{\Lambda}\left(N_{\Delta}^{k}\right) \leqq k ! \rho_{\Lambda}\left(\exp \left(N_{\Delta}\right)\right) \leqq c k !
$$

by Theorem II.3.1. Applying (2.3.2) and (2.3.3) to (2.3.1) we proved the proposition.

We next discuss the thermodynamic limit of the finite volume Gibbs states $\rho_{\Lambda}$. We introduce the CCR algebra of the local observables. For the details we refer to reference [2]. For $f \in L^{2}\left(\Lambda, d^{v} x\right)$, let $a(f)$ and $a(f)^{*}$ be the annihilation and creation operators defined on $\mathscr{F}^{(s)}(\Lambda)$. Then $\Phi(f)=\frac{1}{\sqrt{2}}\left(a(f)+a(f)^{*}\right)$, for real $f$, has a self-adjoint extension, which we write again $\Phi(f)$. We write the Weyl operators by $W(f)=\exp (i \Phi(f))$, and let $\mathscr{U}(\Lambda)$ be the $C^{*}$-algebra generated by the Weyl operator $W(f), f \in L^{2}\left(\Lambda, d^{v} x\right)$, and let

$$
\mathscr{U}=\overline{\bigcup_{\Lambda \in \mathbb{R}^{v}} \mathscr{U}(\Lambda)}
$$

be the quasi local CCR algebra in the sense of [2]. As a consequence of Proposition II.3.2 we have:

Theorem II.3.3. Consider an interacting Bose particle for which the interaction satisfies either Assumption $A$ (with $v<4$ ) or else Assumption $A^{\prime}$. Let $\rho_{A}$ denote the finite volume Gibbs states for some $\beta \in(0, \infty)$ and $\mu \in \mathbb{R}$, and let $\left\{\rho_{\Lambda_{\alpha}}\right\}$ be a subnet such that

$$
\rho(A)=\lim _{\alpha} \rho_{\Lambda_{\alpha}}(A)
$$

exist for all $A \in \bigcup_{\Lambda} \mathscr{U}(\Lambda)$. Then $\rho$ defines an entire analytic state over the CCR algebra $\mathscr{U}$. The state $\rho$ has finite local particle density and hence is locally normal. Proof. We adapt the method used in the proof of Theorem 6.3.22 of [2]. We note that for $\Phi(f)$, supp $f \subset B \subset \Lambda$, and for any $f \in \mathscr{F}^{(s)}(\Lambda)$

$$
\begin{aligned}
\left\|a(f)\left(N_{B}+1\right)^{-1 / 2}\right\| & \leqq\|f\|_{2}, \\
\left\|a(f)^{*}\left(N_{B}+1\right)^{-1 / 2}\right\| & \leqq\|f\|_{2},
\end{aligned}
$$


and

$$
\begin{aligned}
{\left[N_{B}, a(f)\right] } & =-a(f), \\
{\left[N_{B}, a(f)^{*}\right] } & =a(f)^{*} .
\end{aligned}
$$

See reference [2]. Using the above relation it is easy to check that for $L^{2}$-functions $f_{i}$ with compact supports, supp $f_{i} \subset B_{i} \subset \Lambda$,

$$
\begin{aligned}
\rho_{\Lambda}\left(\prod_{i=1}^{k} \Phi\left(f_{i}\right)\right) & \leqq \rho_{\Lambda}\left(\prod_{i=1}^{k}\left(N_{B_{\imath}}+1\right)^{1 / 2}\right) \prod_{i=1}^{k}\|f\|_{2} \\
& \leqq c(k !)^{1 / 2} \prod_{i=1}^{k} N^{+}\left(B_{i}\right)\left\|f_{i}\right\| .
\end{aligned}
$$

Following the procedure used in the proof of Theorem 6.3.22 of [2] and using the bound (2.3.4), we have shown the theorem. For the details of the proof, we refer to $[2]$.

Let $\Phi$ be a real valued function satisfying the estimate: For some $\varepsilon>0$

$$
\int d^{v} x\left(1+|x|^{2}\right)^{v+\varepsilon}|\Phi(x)|^{2}<\infty .
$$

Then the following result follows from Proposition II.3.2. and the same method as in the proof of Theorem 6.3.31 of [2].

Theorem II.3.4. Let $\Phi$ be a positive function satisfying Assumption $A^{\prime}$ and(2.3.4), and let $\rho_{A}$ be the finite volume Gibbs state for the interaction given by the potential $\Phi$. Let $\rho$ be any weak*-limit of $\rho_{\Lambda}$ as $\Lambda \rightarrow \mathbb{R}^{v}$. Then $\rho$ is a modular state.

Since the proof of the theorem is the same as the proof of Theorem 6.3.31 of [2], we do not repeat the proof and refer to [2].

\section{The Wiener Integral Formalism}

\section{III.1. The Wiener Integrals}

We first review the Wiener integral formalism in quantum statistical mechanics for interacting boson particles. For the details we refer to $[2,6,7]$. In the remainder of this paper we will use the following notation:

$$
\begin{aligned}
(x)_{n} & =\left(x_{1}, x_{2}, \ldots, x_{n}\right), \quad x_{i} \in \mathbb{R}^{v}, \\
d(x)_{n} & =\prod_{i=1}^{n} d^{v} x_{i} .
\end{aligned}
$$

The path space of the Wiener measure can be chosen to be

$$
\Omega=\underset{[0, \beta]}{\mathrm{X}} \dot{\mathbb{R}}^{v}
$$

where $\mathbb{R}^{v}$ is the one point compactification of $\mathbb{R}^{v}$. The Wiener measure $P^{\beta}(x, y ; d \omega)$, conditioned on those paths $\omega \in \Omega$ with $\omega(0)=x, \omega(\tau=\beta)=y$, is $\sigma$-additive, finite measure on $\Omega$. It is the path space measure of the process with transition function 
$\exp \left[\frac{1}{2} t \Delta\right]$. The kernel of $\exp \left[\frac{1}{2} t \Delta\right]$ is denoted by $p^{t}(x, y)$ :

$$
p^{t}(x, y)=\frac{1}{(2 \pi t)^{v / 2}} \exp \left[-\frac{|x-y|^{2}}{2 t}\right],
$$

where $|x-y|$ is the Euclidean distance between $x$ and $y$. We have

$$
p^{\beta}(x, y)=\int_{\Omega} P^{\beta}(x, y ; d \omega) .
$$

For any Borel subset $B \subset \mathbb{R}^{\nu}$, let $\chi_{B}^{\beta}$ be the characteristic function of the subset $\{\omega \in \Omega: \omega(\tau) \in B$ for all $\tau \in[0, \beta]\}$. We will drop the superscript $\beta$ from $\chi_{B}^{\beta}$ if there is no confusion involved. We set

$$
\begin{aligned}
P_{\Lambda}^{\beta}(x, y ; d \omega) & \equiv \chi_{\Lambda}(\omega) P^{\beta}(x, y ; d \omega), \\
P_{\Lambda}^{\beta}\left((x)_{n},(y)_{n} ; d(\omega)_{n}\right) & \equiv \prod_{j=1}^{n} P_{\Lambda}^{\beta}\left(x_{j}, y_{j} ; d \omega_{j}\right) .
\end{aligned}
$$

Then $P_{A}^{\beta}(x, y ; d \omega)$ is the path space measure of the process with transition function $\exp \left[\frac{1}{2} t \Delta_{\Lambda}\right]$, where $\Delta_{\Lambda}$ is the Laplacian with 0 -Dirichlet data at $\partial / \Lambda$.

Let $\psi_{\Lambda}^{\beta}\left((x)_{n} ;(y)_{n}\right)$ be the kernel of the operator $\exp \left[-\beta H_{A}^{(n)}\right]$ on $L^{2}\left(\Lambda^{n}\right)$, where $H_{\Lambda}^{(n)}$ is the $n$-particle Hamiltonian given in (2.1.4). By the Feymann-Kac formula. see e.g. $[2,6]$,

$$
\psi_{\Lambda}^{\beta}\left((x)_{n} ;(y)_{n}\right)=\int_{\Omega_{n}} P_{\Lambda}^{\beta}\left((x)_{n},(y)_{n} ; d(\omega)_{n}\right) \exp \left[-\int_{0}^{\beta} U\left((\omega(\tau))_{n}\right) d \tau\right] .
$$

Let $S_{n}$ be the group of the permutations of $\{1,2, \ldots, n\}$ and let $A$ be the multiplication operator by a function $A\left((x)_{n}\right)$ invariant under any $\pi \in S_{n}$. Then we have

$$
\begin{aligned}
& \operatorname{Tr}_{\mathscr{H}_{n}^{(s)}(\Lambda)}\left(A \exp \left[-\beta H_{\Lambda}^{(n)}\right]\right) \\
& \quad=\frac{1}{n !} \sum_{\pi \in S_{n}} \int_{\Lambda_{n}} d(x)_{n} A\left((x)_{n}\right) \int_{\Omega_{n}} P_{\Lambda}^{\beta}\left((x)_{n}, \pi(x)_{n} ; d(\omega)_{n}\right) \exp \left[-\int_{0}^{\beta} U\left((\omega(\tau))_{n} d \tau\right],\right.
\end{aligned}
$$

where $\pi(x)_{n}=\left(x_{\pi(1)}, x_{\pi(2)}, \ldots, x_{\pi(n)}\right)$.

\section{III.2. Some Estimates on the Wiener Integrals}

We collect here basic estimates which will be used later. Let $A \subset \mathbb{R}^{v}$ be a compact subset. We write

$$
\begin{aligned}
P(x, y ; t) & =\int P^{t}(x, y ; d \omega), \\
P_{A}(x, y, ; t) & =\int P^{t}(x, y ; d \omega) \chi_{A}(\omega),
\end{aligned}
$$

and

$$
W_{A}(x, y, ; t)=P(x, y ; t)-P_{A}(x, y ; t)=\int P^{t}(x, y ; d \omega)\left[1-\chi_{A}(\omega)\right] .
$$

We note that for fixed $y \in A$ (or for fixed $x \in A$ ) $W_{A}(x, y ; t)$ is a solution of the diffusion 
equation

$$
\frac{\partial u}{\partial t}=\frac{1}{2} \Delta u
$$

with initial condition $u(x, 0)=0, x \in A$ and boundary condition $u(x, t)=P(x, y ; t)$ $x \in \partial A, t>0$. From the maximum principle we have the following (see Theorem 6.3 .8 of [2] and its proof):

Lemma III.2.1. Let $A \subset \mathbb{R}^{v}$ be a compact subset whose boundary $\partial A$ is a $C^{3}$-surface. Then for all $x, y \in A, 0 \leqq t \leqq \beta$

$$
0 \leqq W_{A}(x, y: t) \leqq e^{v / 2}(2 \pi t)^{-v / 2} \exp \left[-\frac{1}{4 t}\left(d(x, \partial \Lambda)^{2}+d(y, \partial \Lambda)^{2}\right)\right]
$$

where $d(x, \partial \Lambda)$ is the distance between $x$ and $\partial \Lambda$.

Remark. Notice that the right-hand side of the above inequality is independent of the compactness of $A$ and also the smoothness of $\partial A$. Thus, by an approximation argument the inequality holds for any regular subset $A \subset \mathbb{R}^{v}$ with piecewise smooth boundary $\partial A$.

For a unit cube $\Delta$ and for $l \geqq 0$, we write

$$
\Lambda(\Delta, l)=\left\{x \in \mathbb{R}^{v}: \max _{1 \leqq i \leqq v} \inf _{y \in \Delta}\left|x^{i}-y^{i}\right| \leqq l\right\} .
$$

That is, $\Lambda(\Delta, l)$ is the cube with its volume $(2 l+1)^{v}$ containing $\Delta$ at the center. Let us denote

$$
\begin{aligned}
\mathscr{E}_{\Delta, l}= & \left\{\omega \in \Omega: \omega(\tau) \in \Delta, \omega\left(\tau^{\prime}\right) \in \Lambda(\Delta, l+1) \backslash \Lambda(\Delta, l) \text { for some } \tau, \tau^{\prime} \in[0, \beta],\right. \\
& \text { and } \left.\omega\left(\tau^{\prime \prime}\right) \notin \Lambda(\Delta, l+1)^{c} \text { for any } \tau^{\prime \prime} \in[0, \beta]\right\}, \\
\mathscr{E}_{\Delta,-1}= & \{\omega \in \Omega: \omega(\tau) \in \Delta \text { for any } \tau \in[0, \beta]\},
\end{aligned}
$$

where $A^{c}$ is the complement of $A$. Note that any path in $\mathscr{E}_{\Delta, l}, l \geqq 0$, must visit $\Delta$ and $\Lambda(\Delta, l+1) \backslash \Lambda(\Delta, l)$, but not $\Lambda(\Delta, l+1)^{c}$. We define

$$
\begin{aligned}
\mathbb{K}_{\Delta, l}(x, y) & \equiv \int_{\mathscr{E}_{\Delta, l}} P^{\beta}(x, y ; d \omega) \\
& =\int_{\Omega} P^{\beta}(x, y ; d \omega) \chi_{\Lambda(\Delta, l+1)}(\omega)\left[1-\chi_{\Lambda(\Delta, l)}(\omega)\left[1-\chi_{\Delta^{c}}(\omega)\right] .\right.
\end{aligned}
$$

The second equality of (3.2.5) follows from the definition of $\mathscr{E}_{\Delta, l}$ and an easy observation. Let $P_{\Lambda(\Delta, l)}$ be the projection operator onto $L^{2}(\Lambda(\Delta, l)) \subset L^{2}\left(\mathbb{R}^{v}\right)$, and let

$$
\overline{\mathbb{K}}_{\Delta, l} \equiv P_{\Lambda(\Delta, l+1)} \exp \left[\frac{\beta}{4} \Delta\right] P_{\Lambda(\Delta, l+1)}
$$

We write the integral kernel of $\overline{\mathbb{K}}_{\Delta, l}$ by $\overline{\mathbb{K}}_{\Delta, l}(x, y)$ :

$$
\overline{\mathbb{K}}_{\Delta, l}(x, y)=\chi_{\Lambda(\Delta, l+1)} x \int P^{\beta / 2}(x, y ; d \omega) \chi_{\Lambda(\Delta, l+1)}(y) .
$$


For $l=-1$, we write

$$
\begin{aligned}
& \mathbb{K}_{\Delta,-1}(x, y)=\int_{\mathscr{E}_{\Delta,-1}} P^{\beta}(x, y ; d \omega)=\int P^{\beta}(x, y ; d \omega) \chi_{\Delta}(\omega), \\
& \mathbb{\mathbb { K }}_{\Delta,-1}(x, y)=\chi_{\Delta}(x) \int P^{\beta / 2}(x, y ; d \omega) \chi_{\Delta}(y),
\end{aligned}
$$

and let $\overline{\mathbb{K}}_{\Delta,-1}$ be the operator corresponding to the kernel $\overline{\mathbb{K}}_{\Delta,-1}(x, y)$.

Proposition III.2.2. For $l=-1,0,1,2, \ldots$ there are constants $c_{1}>0$ and $c_{2}>0$ such that

a) $\mathbb{K}_{\Delta, l}(x, y) \leqq c_{1} \exp \left(-\frac{l}{16 \beta}\right) \overline{\mathbb{K}}_{\Delta, l}(x, y)$,

b) $\operatorname{Tr}_{L^{2}\left(\mathbb{R}^{y}\right)}\left(\overline{\mathbb{K}}_{\Delta, l}\right) \leqq c_{2}|\Lambda(\Delta, l+1)|$,

where $|\Lambda(\Delta, l+1)|$ is the volume of $\Lambda(\Delta, l+1)$.

Proof (a) We first note that from (3.1.2) and (3.1.3)

$$
\left[\int P^{\beta}(x, y ; d \omega)\right]^{1 / 2}=\left(\frac{\pi \beta}{4}\right)^{v / 4} \int P^{\beta / 2}(x, y ; d \omega) .
$$

Using (3.2.5) and Lemma III.2.1 (and the remark below that) we have

$$
\begin{aligned}
\mathbb{K}_{\Delta, l}(x, y)= & \chi_{\Lambda(\Delta, l / 2)}(x) \mathbb{K}_{\Delta, l}(x, y)+\chi_{\Lambda(\Delta, l / 2)}(x) \mathbb{K}_{\Delta, l}(x, y) \\
\leqq & \chi_{\Lambda(\Delta, l / 2)}(x) \int P^{\beta}(x, y ; d \omega)\left[1-\chi_{\Lambda(\Delta, l)}(\omega)\right] \\
& +\chi_{\Lambda(\Delta, l / 2)} c(x) \int P^{\beta}(x, y ; d \omega)\left[1-\chi_{\Delta^{c}}(\omega)\right] \\
\leqq & c \exp \left(-\frac{l^{2}}{8 \beta}\right)
\end{aligned}
$$

and

$$
\begin{aligned}
\left(\mathbb{K}_{\Delta, l}(x, y)\right)^{1 / 2} & \leqq\left(\int P^{\beta}(x, y ; d \omega) \chi_{\Lambda(\Delta, l+1)}(\omega)\right)^{1 / 2} \\
& \leqq\left(\chi_{\Lambda(\Delta, l+1)}(x) \int P^{\beta}(x, y ; d \omega) \chi_{\Lambda(\Delta, l+1)}(y)\right)^{1 / 2} \\
& \leqq c \overline{\mathbb{K}}_{\Delta, l}(x, y) .
\end{aligned}
$$

Here we have used (3.2.9) and (3.2.7) to get the last inequality. For $l \geqq 0$, the proposition follows from (3.2.10) and (3.2.11). For $l=-1$, the proposition follows from (3.2.9).

(b) Using (3.1.2) and (3.1.3) we have

$$
\operatorname{Tr}_{L^{2}\left(\mathbb{R}^{v}\right)}\left(\overline{\mathbb{K}}_{\Delta, l}\right)=\int_{\Lambda(\Delta, l+1)} d^{v} x \int P^{\beta / 2}(x, x ; d \omega)=\frac{1}{(\pi \beta)^{v / 2}}|\Lambda(\Delta, l+1)| .
$$

This proves the proposition. 
Let $S_{n}$ be the group of permutations of $\{1,2, \ldots, n\}$. As before we write

$$
\begin{gathered}
\mathscr{H}_{n}(\Lambda)=\bigotimes_{i=1}^{n} L^{2}\left(\Lambda, d^{v} x_{i}\right), \\
\mathscr{H}_{n}^{(s)}(\Lambda)=\bigotimes_{i=1}^{n}{ }_{\substack{s\\
}}^{2}\left(\Lambda, d^{v} x_{i}\right) .
\end{gathered}
$$

Let $A_{n} \subset S_{n}$. The subset $A_{n}$ is not a necessary subgroup of $S_{n}$. Let $\left\{f_{i}\right\}$ be an orthonormal base for $L^{2}\left(\Lambda, d^{v} x\right)$, and let $\mathscr{H}_{A_{n}}^{(s)}(\Lambda)$ be the subspace of $\mathscr{H}_{n}(\Lambda)$ spanned by the following vectors:

$$
\operatorname{card}\left(A_{n}\right)^{-1} \sum_{\pi \in A_{n}} f_{i_{1}}\left(x_{\pi(1)}\right) f_{i_{2}}\left(x_{\pi(2)}\right) \ldots f_{i_{n}}\left(x_{\pi(n)}\right)
$$

where card $\left(A_{n}\right)$ is the cardinal number of $A_{n}$. Let $P\left(A_{n}\right)$ be the projection operator onto $\mathscr{H}_{A_{n}}^{(s)}(\Lambda)$. For any $f \in \mathscr{H}_{n}(\Lambda)$ we have

$$
\left(P\left(A_{n}\right) f\right)(x)_{n}=\operatorname{card}\left(A_{n}\right)^{-1} \sum_{\pi \in A_{n}} f\left(\pi(x)_{n}\right) .
$$

If $A$ is an operator of the trace class on $\mathscr{H}_{n}(\Lambda)$ which admits an integral kernel $A\left((x)_{n},(y)_{n}\right)$, it follows from (3.3.1) that $P\left(A_{n}\right) A P\left(A_{n}\right)$ admits the following kernel:

$$
P\left(A_{n}\right) A P\left(A_{n}\right)\left((x)_{n},(y)_{n}\right)=\operatorname{card}\left(A_{n}\right)^{-2} \sum_{\pi, \pi^{\prime} \in A_{n}} A\left(\pi^{-1}(x)_{n}, \pi^{\prime-1}(y)_{n}\right),
$$

and so we have

$$
\operatorname{Tr}_{\mathscr{H}_{n}(A)}\left(P\left(A_{n}\right) A P\left(A_{n}\right)\right)=\operatorname{card}\left(A_{n}\right)^{-2} \sum_{\pi, \pi^{\prime} \in A_{n}} \int_{\Lambda^{n}} d(x)_{n} A\left(\pi^{-1}(x)_{n}, \pi^{\prime-1}(x)_{n}\right) .
$$

The following is obvious: If $A$ is positive operator.

$$
\operatorname{Tr}_{\mathscr{H}_{n}(A)}\left(P\left(A_{n}\right) A P\left(A_{n}\right)\right) \leqq \operatorname{Tr}_{\mathscr{H}_{n}(A)}(A)
$$

In Sect. IV.3 we will consider the kernel of operator $A P\left(A_{n}\right)$. Using (3.3.1) it is easy to derive that

$$
\left(A P\left(A_{n}\right)\right)\left((x)_{n},(y)_{n}\right)=\operatorname{card}\left(A_{n}\right)^{-1} \sum_{\pi \in A_{n}} A\left((x)_{n}, \pi^{-1}(y)_{n}\right) .
$$

In the applications in the following section, we will frequently take $\Lambda=\mathbb{R}^{v}$.

\section{Bounds on Local Number Operators}

\section{IV.1. The Expansions}

In order to show Theorem II.3.1 we employ an expansion method similar to Ruelle's method [13]. Some modifications are necessary to control quantum statistics and fluctuations of paths. We first express the expectation of local number operators in terms of the Wiener integrals. From (2.1.1)-(2.1.5), (2.1.7), (2.1.8) it 
follows that

$$
\begin{aligned}
\Xi_{\Lambda} & =\sum_{n=0}^{\infty} z^{n} \operatorname{Tr}_{\mathscr{H}_{n}^{(s)}(\Lambda)}\left(\exp \left[-\beta H_{\Lambda}^{(n)}\right]\right), \\
\rho_{\Lambda}\left(e^{\alpha N} B\right) & =\Xi_{\Lambda}^{-1} \sum_{n=0}^{\infty} z^{n} \operatorname{Tr}_{\mathscr{H}_{n}^{(s)}(\Lambda)}\left(e^{|\alpha| N_{B}} \exp \left[-\beta H_{\Lambda}^{(n)}\right]\right),
\end{aligned}
$$

where $z=e^{\beta \mu}$ and the term corresponding to $n=0$ equals 1 . In the rest of this paper we use the following notations:

$$
\begin{aligned}
U\left((\omega)_{n}\right) & =\int_{0}^{\beta} U\left((\omega(\tau))_{n}\right) d \tau, \\
N_{B}\left((x)_{n}\right) & =\sum_{i=1}^{n} \chi_{B}\left(x_{i}\right), \quad x_{i} \in \mathbb{R}^{v} .
\end{aligned}
$$

From (3.1.6) it then follows that

$$
\begin{aligned}
& \operatorname{Tr}_{\mathscr{H}_{n}^{(s)}(\Lambda)}\left(e^{|\alpha| N_{B}} \exp \left[-\beta H_{\Lambda}^{(n)}\right]\right) \\
& \quad=\frac{1}{n !} \sum_{\pi \in S_{n}} \int_{\Lambda_{n}} d(x)_{n} e^{|\alpha| N_{B}\left((x)_{n}\right)} \int_{\Omega_{n}} P_{\Lambda}^{\beta}\left((x)_{n}, \pi(x)_{n} ; d(\omega)_{n}\right) e^{-U\left((\omega)_{n}\right)} .
\end{aligned}
$$

Since $B$ can be moved to any place by a translation ( $\Lambda$ will be also moved), one may assume that $B$ is contained in the ball of diameter diam $(B)$ centered at the origin. We will also take $\lambda=1$ in (2.2.1) to avoid non-essential complications arising for $\lambda \neq 1$ in the proof of Theorem II.3.1. Thus $Q(r)$ is the unit cube centered at $r \in Z^{v}$. If superstable interactions satisfy (2.2.2) with $\lambda \neq 1$, we replace unit cubes $Q(r)$ by the cubes having the length of each side $\lambda$. Then the proof for $\lambda \neq 1$ is the same as that for $\lambda=1$ with some trivial modifications.

We will use the following notations:

$$
\begin{aligned}
& Q(r)=\left\{x \in \mathbb{R}^{v}:\left(r^{i}-\frac{1}{2}\right) \leqq x^{i}<\left(r^{i}+\frac{1}{2}\right), r \in Z^{v}\right\}, \\
&|r|=\sup _{1 \leqq i \leqq v}\left|r^{i}\right|, r \in Z^{v}, \\
& \Lambda_{q}=\bigcup_{|r| \leqq q} Q(r), q=0,1,2, \ldots, \\
&\left|\Lambda_{q}\right|=(2 q+1)^{v}: \text { the volume of } \Lambda_{q}, \\
& p_{0}: \text { a fixed natural number such that } B \subset \Lambda_{p_{0}} .
\end{aligned}
$$

For any given path configuration $\left\{\omega_{i}\right\}$, let $n(r, \tau)$ be the number of $\omega_{i}, i=1,2, \ldots, n$, such that $\omega_{i}(\tau) \in Q(r)$. We decompose the space of path configurations $\left\{\omega_{i}\right\}$ into a union of mutually disjoint subsets

$$
\Omega^{n}=\mathscr{E}_{0} \cup\left(\bigcup_{q \geqq p_{0}} \mathscr{E}_{q}\right)
$$

as follows: For fixed $p>l>1$ which we will choose later, let 
$\mathscr{E}_{0}$ : the subset of paths $\left\{\omega_{i}: i=1,2, \ldots, n\right\}$ satisfying

$$
\sup _{\tau \in[0, \beta]} \sum_{r \in \Lambda_{p_{0}}} n(r, \tau)^{p}<\left|\Lambda_{p_{0}}\right|^{l}
$$

$\mathscr{E}_{q}$ : the subset of paths $\left\{\omega_{i}: i=1,2, \ldots, n\right\}$ satisfying

$$
\sup _{\tau \in[0, \beta]} \sum_{r \in \Lambda_{q}} n(r, \tau)^{p} \geqq\left|\Lambda_{q}\right|^{l},
$$

and for all $q^{\prime}>q$

$$
\sup _{\tau \in[0, \beta]} \sum_{r \in \Lambda_{q}} n(r, \tau)^{p}<\left|\Lambda_{q^{\prime}}\right|^{l} .
$$

The above is the first decomposition of $\Omega^{n}$ into disjoint subsets.

For given $q \geqq p_{0}$ we further decompose $\mathscr{E}_{q}$ into a union of subsets as follows. Since, if $\mathscr{E}_{q}$ is empty, $\mathscr{E}_{q}$ does not contribute in the following estimates, one may assume that $\mathscr{E}_{q}$ is not empty. Let $K$ be a subset of $\{1,2, \ldots, n\}$ and let $n_{K}(r, \tau)$ be the number of $\omega_{i}$ 's, $i \in K$, such that $\omega_{i}(\tau) \in Q(r)$. For any configuration of $n$-paths in $\mathscr{E}_{q}$, there is at least one smallest subset $K \subset\{1,2, \ldots, n\}$ satisfying the following condition:

$$
\sup _{\tau \in[0, \beta]} \sum_{r \in \Lambda_{q}} n_{K}(r, \tau)^{p}=\sup _{\tau \in[0, \beta]} \sum_{r \in \Lambda_{q}} n(r, \tau)^{p},
$$

and for any $K^{\prime} \subset\{1,2, \ldots, n\}$ with card $\left(K^{\prime}\right)<\operatorname{card}(K)$,

$$
\sup _{\tau \in[0, \beta]} \sum_{r \in \Lambda_{q}} n_{K^{\prime}}(r, \tau)^{p}<\sup _{\tau \in[0, \beta]} \sum_{r \in \Lambda_{q}} n_{K}(r, \tau)^{p} .
$$

We define

$$
\begin{aligned}
\mathscr{E}_{q, k}=\left\{\left\{\omega_{i}\right\} \in \mathscr{E}_{q}:\right. & \text { There exists a } K \text { satisfying (4.1.9) } \\
& \text { and (4.1.10) with } \operatorname{card}(K)=k\} .
\end{aligned}
$$

We remark that the paths $\omega_{i}, i \in K$, must visit $\Lambda_{q}$ at least one time at $\tau_{0} \in[0, \beta]$ simultaneously by the definition of $K$. We have

$$
\mathscr{E}_{q}=\bigcup_{k} \mathscr{E}_{q, k}
$$

From (4.1.7) and (4.1.8) it follows that

$$
\left|\Lambda_{q}\right|^{l / p} \leqq k \leqq\left|\Lambda_{q+1}\right|^{1+(l-1) / p} .
$$

The first inequality follows from Lemma IV.4.2(a) in the next section and the second inequality follows from (4.1.8) and the Hölder inequality:

$$
\sum_{r \in \Lambda_{q+1}} n(r, \tau) \leqq\left|\Lambda_{q+1}\right|\left(\left|\Lambda_{q+1}\right|^{-1} \sum_{r \in \Lambda_{q+1}} n(r, \tau)^{p}\right)^{1 / p} \leqq\left|\Lambda_{q+1}\right|^{1+(l-1) / p} \text { on } \mathscr{E}_{q}
$$

Thus, from (4.1.1), (4.1.3) and (4.1.12) we have

$$
\rho_{\Lambda}\left(e^{\alpha N_{B}}\right) \leqq G_{0}+\sum_{n=0}^{\infty} \sum_{q \geqq p_{0}} \sum_{k \geqq\left|\Lambda_{q}\right|^{l / p}} G_{q, k}^{(n)},
$$


where $G_{0}$ is the contribution from $\mathscr{E}_{0}$ :

$$
G_{0}=\Xi_{\Lambda}^{-1} \sum_{n=1}^{\infty} \frac{z^{n}}{n !} \sum_{\pi \in S_{n}} \int_{A^{n}} d(x)_{n} e^{|\alpha| N_{B}\left((x)_{n}\right)} \int_{\mathscr{E}_{0}} P_{\Lambda}^{\beta}\left(\left(x_{n}\right), \pi(x)_{n} ; d(\omega)_{n} e^{-U\left((\omega)_{n}\right)},\right.
$$

and

$$
G_{q, k}^{(n)}=\Xi_{\Lambda}^{-1} \frac{z^{n}}{n !} \sum_{\pi \in S_{n}} \int_{A n} d(x)_{n} e^{|\alpha| N_{B}\left((x)_{n}\right)} \int_{\mathscr{E}_{q, k}} P_{\Lambda}^{\beta}\left((x)_{n}, \pi(x)_{n} ; d(\omega)_{n}\right) e^{-U\left((\omega)_{n}\right)} .
$$

Since, by (4.1.13)

$$
N_{B}\left((x)_{n}\right) \leqq \sum_{r \in \Lambda_{q+1}} n(r, \tau=0) \leqq\left|\Lambda_{q+1}\right|^{1+(l-1) / p} \quad \text { on } \mathscr{E}_{q} .
$$

We have

$$
G_{0}=\exp \left[|\alpha|\left|\Lambda_{p_{0}}\right|^{1+(l-1) / p}\right]
$$

and

$$
G_{q, k}^{(n)} \leqq \Xi_{\Lambda}^{-1} z^{n} \exp \left[|\alpha|\left|\Lambda_{q+1}\right|^{1+(l-1) / p}\right] \bar{G}_{q, k}^{(n)},
$$

where

$$
\bar{G}_{q, k}^{(n)} \equiv \frac{1}{n !} \sum_{\pi \in S_{n}} \int_{\Lambda^{n}} d(x)_{n} \int_{\mathscr{E} q, k} P_{\Lambda}^{\beta}\left((x)_{n}, \pi(x)_{n} ; d(\omega)_{n}\right) e^{-U\left((\omega)_{n}\right)} .
$$

The problem is now reduced to obtain uniform bounds of (4.1.20) so that the expression in (4.1.19) is summable.

\section{Basic Ideas}

We recall that for any configuration of paths $\left\{\omega_{i}\right\}$ in $\mathscr{E}_{q, k}$ there is a subset $K \subset\{1,2, \ldots, n\}$ with $\operatorname{card}(K)=k$ such that (4.1.9) and (4.1.10) hold. There are $n ! /(n-k) ! k$ ! ways to choose $k$-paths among $n$-paths. If one reindexes $k$-paths $\omega_{i}$, $i \in K$, so that $K=\{1,2, \ldots, k\}$, and if one defines

$$
\overline{\mathscr{E}}_{q, k}=\left\{\left\{\omega_{i}\right\} \in \mathscr{E}_{q}: K=\{1,2, \ldots, k\} \text { satisfies (4.1.9) and (4.1.10) }\right\},
$$

it follows from $(4.1 .20)$ that

$$
\bar{G}_{q, k}^{(n)}=\frac{1}{(n-k) ! k !} \sum_{\pi \in S_{n}} \int_{A^{n}} d(x)_{n} \int_{\mathscr{E} q, k} P_{A}^{\beta}\left((x)_{n}, \pi(x)_{n} ; d(\omega)_{n}\right) e^{-U\left((\omega)_{n}\right)} .
$$

Notice that we have an $(n-k)$ ! factor instead of $n$ !. The total number of terms in the above is $\operatorname{card}\left(S_{n}\right)=n$ !. This is the main problem arising from quantum statistics.

For any configuration $\left\{\omega_{i}\right\}$ of paths in $\overline{\mathscr{E}}_{q, k}$ we write

$$
\begin{aligned}
U_{1}\left((\omega)_{k}\right) & \equiv \sum_{1 \leqq i<j \leqq k} \int_{0}^{\beta} \Phi\left(\omega_{i}(\tau)-\omega_{j}(\tau)\right) d \tau \equiv \int_{0}^{\beta} U_{1}\left((\omega(\tau))_{k} d \tau,\right. \\
U_{2}\left((\omega)_{n-k}\right) & \equiv \sum_{k+1 \leqq i<j \leqq n} \int_{0}^{\beta} \Phi\left(\omega_{i}(\tau)-\omega_{j}(\tau)\right) d \tau \equiv \int_{0}^{\beta} U_{2}\left((\omega(\tau))_{n-k}\right) d \tau, \\
W\left((\omega)_{k},(\omega)_{n-k}\right) & \equiv \sum_{i=1}^{k} \sum_{j=k+1}^{n} \int_{0}^{\beta} \Phi\left(\omega_{i}(\tau)-\omega_{j}(\tau)\right) d \tau \equiv \int_{0}^{\beta} W\left((\omega(\tau))_{k},(\omega(\tau))_{n-k}\right) d \tau .
\end{aligned}
$$


Then

$$
U\left((\omega)_{n}\right)=U_{1}\left((\omega)_{k}\right)+W\left((\omega)_{k},(\omega)_{n-k}\right)+U_{2}\left((\omega)_{n-k}\right) .
$$

From the definition of $\overline{\mathscr{E}}_{q, k}$ in (4.2.1) and from (4.1.9) and (4.1.7) we conclude that there exists $\tau_{0} \in[0, \beta]$ such that

$$
\sum_{r \in \Lambda_{q}} \bar{n}\left(r, \tau_{0}\right)^{p} \geqq\left|\Lambda_{q}\right|^{l} \quad \text { on } \overline{\mathscr{E}}_{q, k},
$$

where $\bar{n}\left(r, \tau_{0}\right)$ be the number of $\omega_{i}$ 's, $i=1,2, \ldots, k$, such that $\omega_{i}\left(\tau_{0}\right) \in Q(r)$.

We now discuss basic ideas briefly. First, one has to control the divergence factors in (4.1.19):

$$
\exp \left[|\alpha|\left|\Lambda_{q+1}\right|^{1+(l-1) / p}\right],
$$

which come from the bounds for $\exp \left[\alpha N_{B}\right]$ on $\mathscr{E}_{q, k}$ (or on $\overline{\mathscr{E}}_{q, k}$ ). We will obtain some convergence factors from the interaction terms $U_{1}\left((\omega)_{k}\right)$. We note that for any configuration $\left\{\omega_{i}\right\}$ of paths in $\overline{\mathscr{E}}_{q, k}$ there exists $\tau_{0} \in[0, \beta]$ such that (4.2.5) holds. From the strong superstability condition in Assumption A in Sect. II.2, one obtains that

$$
\begin{aligned}
U_{1}\left(\left(\omega\left(\tau_{0}\right)\right)_{k}\right) & \geqq \sum_{r}\left[A \bar{n}\left(r, \tau_{0}\right)^{p^{\prime}}-B \bar{n}\left(r, \tau_{0}\right)\right] \\
& \geqq \sum_{r \in \Lambda_{q}} A \bar{n}\left(r, \tau_{0}\right)^{p}-B k,
\end{aligned}
$$

where $k$ is bounded by (4.1.13). Using the Hölder's inequality and (4.2.5) one gets that for $1<l<p<p^{\prime}$

$$
U_{1}\left(\left(\omega\left(\tau_{0}\right)\right)_{k}\right) \geqq A\left|\Lambda_{q}\right|^{1+(l-1) p^{\prime} / p}-B\left|\Lambda_{q+1}\right|^{1+(l-1) / p} .
$$

Thus, if the above inequality holds for any $\tau \in[0, \beta]$, one gets the convergent factors from $\exp \left(-U_{1}\left((\omega)_{k}\right)\right)$. But one does not expect that (4.2.7) holds for all $\tau \in[0, \beta]$, because of the fluctuations of paths $\omega_{i}, i=1,2, \ldots, k$. If the fluctuations are large, one expects the contributions are small in the sense of Lemma III.2.1 and Proposition III.2.2. On the other hand, if the fluctuations are small, one expects that (4.2.7) holds approximately. Thus one may be able to permit a small amount of the fluctuations.

We define the fluctuations of $k$-paths as follows: We write

$$
\begin{aligned}
V(\omega) & =\sup _{\tau_{1}, \tau_{2} \in[0, \beta]}\left|\omega\left(\tau_{1}\right)-\omega\left(\tau_{2}\right)\right|^{2}, \\
V\left((\omega)_{k}\right) & =\sum_{i=1}^{k} V\left(\omega_{i}\right) .
\end{aligned}
$$

The following result which we will prove in Sect. IV.4 shows that if one adds a small fluctuation term one gets convergent factors from the interactions. We write

$$
\gamma\left(v, p^{\prime}\right) \equiv 1+\left(2\left(p^{\prime}-1\right) / v p^{\prime}-(v-2)\right) .
$$

We then have: 
Theorem IV.2.1 a) Let the interactions satisfy Assumption A in Sect. II.2. with $p^{\prime}$ satisfying the inequality

$$
\frac{p^{\prime}}{p^{\prime}-1}<\gamma\left(v, p^{\prime}\right)
$$

Let $p>l>1$ have been chosen such that

(1) $l / p<2 / v$,

(2) $\frac{p^{\prime}}{p^{\prime}-1} \leqq p \leqq \gamma\left(v, p^{\prime}\right)$.

Then for any $b>0, p_{0}$ in (4.1.4) and (4.1.6) can be chosen sufficiently large such that there is a constant $c>0$ independent of $q, k$ and $\Lambda$ such that

$$
U_{1}\left((\omega)_{k}\right)+b V\left((\omega)_{k}\right)+W\left((\omega)_{k}, \omega(\omega)_{n-k}\right) \geqq c\left|\Lambda_{q+1}\right|^{\left[1+\left((l-1) \gamma\left(v, p^{\prime}\right) / p\right)\right]}
$$

on $\overline{\mathscr{E}}_{q, k}$ for $q \geqq p_{0}$.

b) Let the interactions satisfy Assumption $A^{\prime}$ in Sect. II.2, and let $p>l>1$ have been chosen such that $p \leqq \gamma\left(v, p^{\prime}\right)$. Then for any fixed $b>0$ there is a constant $c>0$ independent of $q, k$ and $\Lambda$ such that

$$
U_{1}+b V\left((\omega)_{k}\right) \geqq c\left|\Lambda_{q+1}\right|^{\left[1+\left((l-1) \gamma\left(v, p^{\prime}\right) / p\right)\right]}
$$

holds on $\overline{\mathscr{E}}_{q, k}$ for sufficiently large $p_{0}$.

From the fact that $\gamma\left(v, p^{\prime}\right)>1$ it follows that

$$
1+\frac{l-1}{p}<1+\frac{l-1}{p} \gamma\left(v, p^{\prime}\right)
$$

and so we have convergent factors from the interaction by the above theorem.

Lemma IV.2.2. Under the assumption in Theorem IV.2.1, one can choose $p>l>1$ such that (1) and (2) in the theorem hold for $v \leqq 3$.

Proof. For $v=3$ the assumption implies $p^{\prime}>3$ and so

$$
\frac{p^{\prime}}{p^{\prime}-1}<\frac{3}{2} \text { and } \gamma\left(3, p^{\prime}\right)>\frac{3}{2}
$$

If one chooses $l=1+\varepsilon$ for $\varepsilon>0$, the condition (1) in the theorem implies that $p$ has to satisfy $p>\frac{3}{2}(1+\varepsilon)$. For given $p^{\prime}$ one can choose $\varepsilon$ sufficiently small such that $\frac{3}{2}(1+\varepsilon)<\gamma\left(3, p^{\prime}\right)$. Thus $p$ can be chosen so that (1) and (2) hold. The argument similar to the above gives the proof for $v=1,2$. This completes the proof of the lemma.

If $v \geqq 4$, then $\gamma\left(v, p^{\prime}\right)<1+\frac{2}{v}$ for any $p^{\prime} \geqq 2$. The condition (1) in Theorem IV.2.1 implies $p>\frac{v}{2}$ and so $p>\gamma\left(v, p^{\prime}\right)$. The bound in the theorem will be failed to hold in this case. This is the reason why we require $v<4$. We will discuss the reason in Section $\mathrm{V}$ in more details. 


\section{IV.3. The Proof of the Main Result: Control of Quantum Statistics}

In this section we prove Theorem II.3.1 under the assumption that Theorem IV.2.1 holds. From Theorem IV.2.1, (4.2.2) and (4.2.4), and from the fact that $W\left((\omega)_{k},(\omega)_{n-k}\right) \geqq 0$ for repulsive interactions, it follows that

$$
\left.\bar{G}_{q, k}^{(n)} \leqq\left.\exp |-c| \Lambda_{q+1}\right|^{1+(l-1) \gamma\left(v, p^{\prime}\right) / p}\right] \bar{F}_{q, k}^{(n)},
$$

where

$$
\begin{aligned}
\bar{F}_{q, k}^{(n)} \equiv & \frac{1}{(n-k) ! k !} \sum_{\pi \in S_{n}} \int_{\Lambda^{n}} d(x)_{n} \int_{\tilde{\delta}_{q, k}} P_{\Lambda}^{\beta}\left((x)_{n}, \pi(x)_{n} ; d(\omega)_{n}\right) \\
& \cdot \exp \left[b V\left((\omega)_{k}\right)-U_{2}\left((\omega)_{n-k}\right)\right] .
\end{aligned}
$$

As we have discussed before, there are $n$ ! terms in (4.3.2). Therefore one has to show that many terms in (4.3.2) do not contribute. The main idea is decoupling of the paths in $\left\{\omega_{i}: i=1,2, \ldots, k\right\}$ from the paths in $\left\{\omega_{i} ; i=k+1, \ldots, n\right\}$. On the subset $\overline{\mathscr{E}}_{q, k}$ a path $\omega_{i}, i=1,2, \ldots, k$, may join to a path $\omega_{l}, l=k+1, \ldots, n$, to form a composite path, i.e., $x_{\pi(i)}=x_{l}$. If the fluctuation of $\omega_{i}$ is small, there will not be many ways to form the composite trajectory. On the other hand, if the fluctuation is large, there will be a convergent factor by Proposition III.2.2. Thus we further decompose the subset $\overline{\mathscr{E}}_{q, k}$ into a union of disjoint subsets corresponding to large and small fluctuations. The decomposition is as follow. We remember that $\omega_{i}, i=1,2, \ldots, k$, must visit $\Lambda_{q}$ at least one time. Thus we have

$$
\begin{aligned}
& \int_{\tilde{\mathscr{E}} q, k} P_{\Lambda}^{\beta}\left((x)_{n}, \pi(x)_{n} ; d(\omega)_{n}\right) \ldots \\
& \quad=\int_{\tilde{E}_{q, k}} P_{\Lambda}^{\beta}\left((x)_{n}, \pi(x)_{n} ; d(\omega)_{n}\right) \prod_{i=1}^{k}\left[1-\chi_{\Lambda_{q}^{\mathrm{q}}}(\omega)\right] \ldots
\end{aligned}
$$

where $\Lambda_{q}^{c}$ is the complement of $\Lambda_{q}$. Notice that

$$
\left[1-\chi_{\Lambda_{q}^{c}}(\omega)\right] \leqq \sum_{r \in \Lambda_{q}}\left[1-\chi_{Q(r)}(\omega)\right] .
$$

That is, in order to visit $\Lambda_{q}$ at least one time the path has to visit some $Q(r)$ in $\Lambda_{q}$. We define

$$
\Lambda(\Delta, l), \mathscr{E}_{\Delta, l} \text { and } \mathscr{E}_{\Delta,-1}
$$

as in (3.2.3) and (3.2.4). We also note that

$$
\left[1-\chi_{\Delta c}(\omega)\right]=\left[\chi_{\Delta}(\omega)+\sum_{l=0}^{\infty} \chi_{\Lambda(\Delta, l+1)}(\omega)\left(1-\chi_{\Lambda(\Delta, l)}(\omega)\right)\right]\left[1-\chi_{\Delta^{c}}(\omega)\right],
$$

and

$$
\begin{aligned}
\int_{\mathscr{\delta}_{\Lambda, l}} P_{\Lambda}^{\beta}(x, y ; d \omega) \cdots & =\int P_{\Lambda}^{\beta}(x, y ; d \omega) \chi_{\Lambda(\Delta, l+1)}(\omega)\left[1-\chi_{\Lambda(\Delta, l)}(\omega)\right]\left[1-\chi_{\Delta c}(\omega)\right] \ldots \\
\int_{\mathscr{E}_{\Delta,-1}} P_{\Lambda}^{\beta}(x, y ; d \omega) \cdots & =\int P_{\Lambda}^{\beta}(x, y ; d \omega) \chi_{\Delta}(\omega) \ldots
\end{aligned}
$$


From (4.3.4), (4.3.5) and (4.3.6) one obtains that for any positive function $F$ on $\Omega$

$$
\int P_{\Lambda}^{\beta}(x, y ; d \omega)\left[1-\chi_{\Lambda_{q}^{q}}(\omega)\right] F(\omega) \leqq \sum_{\Delta \subset A_{q}} \sum_{l=-1}^{\infty} \int_{\mathscr{S}_{\Delta, l}} P^{\beta}(x, y ; d \omega) F(\omega),
$$

where $\sum_{\Delta \subset \Lambda_{q}}$ is the summation over unit cubes $\Delta=Q(r) \subset \Lambda_{q}$. Let $\mathscr{E}_{\Delta, l}^{(i)}$ be the subset $\mathscr{E}_{\Delta, l}$ for the $i$-path, $i=1,2, \ldots, k$. We write

$$
\overline{\mathscr{E}}_{q}^{k}\left(\left(\Delta_{1}, l_{1}\right), \ldots,\left(\Delta_{k}, l_{k}\right)\right)=\left\{(\omega)_{n} \in \overline{\mathscr{E}}_{q, k}: \omega_{i} \in \mathscr{E}_{\Delta_{i}, l_{l}}^{(i)}, i=1,2, \ldots, k\right\} .
$$

We note that for any configuration $(\omega)_{n}$ in $\overline{\mathscr{E}}_{q}^{k}\left(\left(\Delta_{1}, l_{1}\right), \ldots,\left(\Delta_{k}, l_{k}\right)\right)$ and for any $i \in\{1,2, \ldots, k\}$,

$$
\sup _{\tau_{1}, \tau_{2} \in[0, \beta]}\left|\omega_{i}\left(\tau_{1}\right)-\omega_{i}\left(\tau_{2}\right)\right|^{2} \leqq \operatorname{diam}\left(\Lambda_{l_{l}+1}\right)^{2} \leqq 4 v\left(l_{i}+2\right)^{2},
$$

where $\operatorname{diam}(A)$ is the diameter of $A$, and so

$$
V\left((\omega)_{k}\right) \leqq 4 v \sum_{i=1}^{k}\left(l_{i}+2\right)^{2}
$$

on $\overline{\mathscr{E}}_{q}^{k}\left(\left(\Delta_{1}, l_{1}\right), \ldots,\left(\Delta_{k}, l_{k}\right)\right)$. We now apply (4.3.3), (4.3.7) and (4.3.9) (in that order) to (4.3.2) to obtain the bound

$$
\begin{aligned}
\bar{F}_{q, k}^{(n)} \leqq & \frac{1}{(n-k) ! k !} \sum_{\Delta_{1} \subseteq A_{q}} \ldots \sum_{\Delta_{k} \subseteq A_{q}} \sum_{l_{1}=-1}^{\infty} \ldots \sum_{l_{k}=-1}^{\infty} \\
& \cdot \exp \left[4 v b \sum_{i=1}^{k}\left(l_{i}+2\right)^{2}\right] \bar{F}_{q}^{k}\left(\left(\Delta_{1}, l_{1}\right), \ldots,\left(\Delta_{k}, 1_{k}\right)\right),
\end{aligned}
$$

where

$$
\begin{gathered}
\bar{F}_{q}^{k}\left(\left(\Delta_{1}, l_{1}\right), \ldots,\left(\Delta_{k}, l_{k}\right)\right)=\sum_{\pi \in S_{n}} \int_{\Lambda^{n}} d(x)_{n} \mathbb{K}\left((x)_{n}, \pi(x)_{n}\right), \\
\mathbb{K}\left((x)_{n},(y)_{n}\right) \equiv \int_{\left.\bar{\delta}^{k}{ }_{q}\left(\Delta_{1}, l_{1}\right), \ldots,\left(\Delta_{k}, l_{k}\right)\right)} P_{\Lambda}^{\beta}\left((x)_{n},(y)_{n} ; d(\omega)_{n}\right) \exp \left[-U_{2}\left((\omega)_{n-k}\right)\right] .
\end{gathered}
$$

We recall that for any configuration of $n$-paths $(\omega)_{n}$ in $\overline{\mathscr{E}}_{q}^{k}\left(\left(\Delta_{1}, l_{1}\right), \ldots,\left(\Delta_{k}, l_{k}\right)\right)$, the path $\omega_{i}, i=1,2, \ldots, k$, must stay inside of $\Lambda\left(\Delta_{i}, l_{i}+1\right)$ by (3.2.4) and (4.3.8). This means that $\omega_{i}, i=1,2, \ldots, k$, cannot form a composite path (trajectory) with any path $\omega_{l}$ if $\omega_{l}(\tau=0) \in \Lambda\left(\Delta_{i}, l_{i}+1\right)^{c}$. According to the bounds in (4.1.14) there are at most

$$
\left|\Lambda_{q+l_{i}+2}\right|^{1+(l-1) / p}
$$

number of paths which can hit $\Lambda\left(\Delta_{i}, l_{i}+1\right) \subset \Lambda_{q+l_{i}+2}$ at $\tau=0$ (or $\tau=\beta$ ). Thus, if $l_{i}, i=1,2, \ldots, k$, are small, many terms in (4.3.11) will vanish. If $l_{i}$ 's are large, there will be convergent factors by Proposition III.2.2. This is the idea of controlling quantum statistics.

We will use the following abbreviated notation:

$$
M\left(l_{i}\right) \equiv\left|\Lambda_{q+l_{i}+2}\right|^{1+(l-1) / p}
$$


We also introduce the following notations:

$S(k, n)$ : the subgroup of $S_{n}$ consisting of the permutations of $\{k+1, k+2, \ldots, n\}$,

$E\left(m, m^{\prime}\right)$ : the subset of $S_{n}$ consisting of the interchanging of $m$ with one of $m, m+1, \ldots, m^{\prime}$ for $m \leqq m^{\prime} \leqq n$.

We make the following convention: $E\left(m, m^{\prime}\right)=E(m, n)$ if $m^{\prime}>n$, and for any $\pi, \sigma \in S_{n}, \pi \sigma$ means that $\sigma$ followed by $\pi ; k \rightarrow \pi(\sigma(k))$. We define

$$
\begin{aligned}
& E_{k}\left(l_{1}, l_{2}, \ldots, l_{k}\right)=\left\{P=P_{k} P_{k-1} \ldots P_{1}: P_{i} \in E\left(i,\left[2 M\left(l_{i}\right)\right]\right) \text { for } i=1,2, \ldots, k\right\}, \\
& \widetilde{E}_{k}\left(l_{1}, l_{2}, \ldots, l_{k}\right)=\left\{P \in S_{n}: P^{-1} \in E_{k}\left(l_{1}, \ldots, l_{k}\right)\right\} \\
& \widetilde{E}_{n, k}=\left\{P=P_{1} P_{2} \ldots P_{k}: P_{i} \in E(i, n) \text { for } i=1,2, \ldots, k\right\} .
\end{aligned}
$$

Here we have used the symbol $[a]$ for the greatest natural number which is not greater than $a$, and $M\left(l_{i}\right)$ has been defined in (4.3.12). We note that

$$
S_{n}=\widetilde{E}_{n, k} S(k, n) \equiv\left\{p \sigma: P \in \widetilde{E}_{n, k}, \sigma \in S(k, n)\right\},
$$

and

$$
\operatorname{card}\left(E_{k}\left(l_{1}, \ldots, l_{k}\right)\right)=\operatorname{card}\left(\tilde{E}_{k}\left(l_{1}, \ldots, l_{k}\right)\right) \leqq \prod_{i=1}^{k} 2 M\left(l_{i}\right) .
$$

For any $\sigma \in S(k, n)$ we will use the following notation:

$\chi_{\sigma}$ : the characteristic function of the subset

$$
\left\{(x)_{n} \in\left(\mathbb{R}^{v}\right)^{n}:\left\|x_{\sigma(k+1)}\right\| \leqq\left\|x_{\sigma(k+2)}\right\| \leqq \cdots \leqq\left\|x_{\sigma(n)}\right\|\right\},
$$

where $\|x\|=\max _{1 \leqq j \leqq v}\left|x^{i}\right|$. We then have

$$
\sum_{\sigma^{\prime} \in S(k, n)} x_{\sigma^{\prime}}\left((x)_{n}\right)=\sum_{\sigma^{\prime} \in S(k, n)} \chi_{e}\left(\sigma^{\prime}(x)_{n}\right)=1,
$$

where $e \in S(k, n)$ is the identity element.

Lemma 4.3.1. $\mathbb{K}\left((x)_{n},(y)_{n}\right)$ be defined as in (4.3.11). Then

$$
\bar{F}_{q}^{k}\left(\left(\Delta_{1}, l_{1}\right), \ldots,\left(\Delta_{k}, l_{k}\right)\right) \leqq k ! \sum_{P \in \tilde{E}_{k}\left(l_{1}, \ldots l_{k}\right)} \sum_{\sigma^{\prime}, \sigma \in S(k, n)} \int_{A^{n}} d(x)_{n} \chi_{e}\left((x)_{n}\right) \mathbb{K}\left(\sigma^{\prime}(x)_{n}, P \sigma(x)_{n}\right) .
$$

Proof. Substituting (4.3.18) into (4.3.11) and doing a change of variables $\left(\sigma^{\prime}(x)_{n} \rightarrow(x)_{n}\right)$, we obtain

$$
\bar{F}_{q}^{k}\left(\left(\Delta_{1}, l_{1}\right), \ldots,\left(\Delta_{k}, l_{k}\right)\right)=\sum_{P \in \widetilde{E}_{n, k}} \sum_{\sigma^{\prime}, \sigma \in S(k, n)} \int_{\Lambda^{n}} d(x)_{n} \chi_{e}\left((x)_{n}\right) \mathbb{R}\left(\sigma^{\prime}(x)_{n}, P \sigma(x)_{n}\right) .
$$

Here we have used the fact the $S_{n}=\widetilde{E}_{n, k} S(k, n)$. Under the assumption that $l_{1} \leqq l_{2} \leqq \cdots \leqq l_{k}$, we will show that the summation over $P \in \widetilde{E}_{n, k}$ in the above can be replaced by the summation over $P \in \widetilde{E}_{k}\left(l_{1}, \ldots, l_{k}\right)$. Then for general $l_{1}, l_{2}, \ldots, l_{k}$, the lemma follows from reindexing $\omega_{1}, \omega_{2}, \ldots, \omega_{k}$ such that $l_{1} \leqq l_{2} \cdots \leqq l_{k}$ in the new indexes. This is the reason why we have $k$ ! in the lemma.

We now assume that $l_{1} \leqq l_{2} \leqq \cdots \leqq l_{k}$. Assume that $P \notin \widetilde{E}_{k}\left(l_{1}, \ldots, l_{k}\right)$ in the 
integrand of (4.3.19). Then there is at least one $P_{i} \notin E\left(i,\left[2 M\left(l_{i}\right)\right]\right)$ such that $P=P_{1} \ldots P_{i} \ldots P_{k}$ and $P_{j} \in E\left(j,\left[2 M\left(l_{j}\right)\right]\right)$ for all $j<i$. Write

$$
P_{i}=\left(\begin{array}{cc}
i & m \\
m & i
\end{array}\right), \quad P_{j}=\left(\begin{array}{cc}
j & m_{j} \\
m_{j} & j
\end{array}\right) .
$$

Then $m>2 M\left(l_{i}\right)$ and $m_{j}<m$ for all $j<i$ by the assumption $l_{j} \leqq l_{i}$ for $j<i$. A simple inspection shows that $P \sigma(i)=P_{1} \ldots P_{i}(i)=P_{1} \ldots P_{i-1}(m)=m$. We recall that by the definition of $\mathbb{K}\left(\sigma^{\prime}(x)_{n}, P \sigma(x)_{n}\right), \omega_{i}(\beta)=x_{P \sigma(i)}=x_{m}$. Since $\omega_{i} \in \mathscr{E}_{\Delta_{i}, l_{i}}^{(i)}$ in the definition of $\mathbb{K}\left((x)_{n},(y)_{n}\right)$ in (4.3.11), $\omega_{i}(\beta) \in \Lambda_{q+l_{i}+2}$, and so $x_{m} \in \Lambda_{q+l_{2}+2}$. Because of the factor $\chi_{e}\left((x)_{n}\right)$ in the integrand in (4.3.19), the integration variables $x_{j}, k+1 \leqq j \leqq m$, should be restricted to $x_{j} \in \Lambda_{q+l_{t}+2}$. This means that at least $m-k$ paths have to pass $\Lambda_{q+l_{2}+2}$ at $\tau=0$ (and also at $\tau=\beta$ ). In the definition of $\mathbb{K}\left((x)_{n},(y)_{n}\right)$ the integration over path configuration space has been restricted to $\overline{\mathscr{E}}_{q}^{k}\left(\left(\Delta_{1}, l_{1}\right), \ldots,\left(\Delta_{k}, l_{k}\right)\right) \subset \mathscr{E}_{q}$, and so more than $M\left(l_{i}\right)$ paths can not pass $\Lambda_{q+l_{1}+2}$ at $\tau=0$ by (4.1.14). We note that $m-k>M\left(l_{i}\right)$ by (4.1.13). Thus $\mathbb{K}\left(\sigma^{\prime}(x)_{n}, P \sigma(x)_{n}\right)$ must be vanished if $P \notin \widetilde{E}_{k}\left(l_{1}, \ldots, l_{k}\right)$. This completes the proof of the lemma.

Let $P(S(k, n))$ and $P\left(E_{k}\left(l_{1}, \ldots, l_{k}\right)\right)$ be the projection operators onto $\mathscr{H}_{S(k, n)}^{(s)}(\Lambda)$ and $\mathscr{H}_{E_{k}\left(l_{1}, \ldots, l_{k}\right)}^{(s)}(\Lambda)$ respectively, which we have introduced in Sect. III.3. For any $\sigma \in S(k, n)$, let $\chi_{\sigma}$ be the (projection) operator defined by $\left(\chi_{\sigma} f\right)(x)_{n}=\chi_{e}\left(\sigma(x)_{n}\right) f\left((x)_{n}\right)$ for any $f \in \mathscr{H}_{n}\left(\mathbb{R}^{v}\right)$. From (4.3.18) we have

$$
\sum_{\sigma \in S(k, n)} \chi_{\sigma}=\mathbb{1}
$$

In order to avoid notational complication we will use the following abbreviated notations:

$$
\begin{aligned}
P_{1} & \equiv P(S(k, n)) \\
P_{2} & \equiv P\left(E_{k}\left(l_{1}, \ldots, l_{k}\right)\right), \\
C_{1.2} & \equiv \operatorname{card}(S(k, n))^{2} \operatorname{card}\left(E_{k}\left(l_{1}, \ldots, l_{k}\right)\right) .
\end{aligned}
$$

Let $\mathbb{K}$ be the operator on $\mathscr{H}_{n}(\Lambda)$ defined by its kernel $\mathbb{K}\left((x)_{n},(y)_{n}\right)$, where $\mathbb{K}\left((x)_{n},(y)_{n}\right)$ has been defined in (4.3.11). We recall the definition of $\widetilde{E}_{k}\left(l_{1}, \ldots, l_{k}\right)$. Using (3.3.2) and (3.3.5) in Sect. III.3, the following is easy to derive:

$$
\begin{aligned}
& \operatorname{Tr}_{\mathscr{H}_{n}(\Lambda)}\left(\chi_{e} P_{1} \mathbb{R} P_{1} P_{2} \chi_{e}\right) \\
& \quad=C_{1,2}^{-1} \sum_{P \in \tilde{E}_{k}\left(l_{1}, \ldots, l_{k}\right)} \sum_{\sigma^{\prime}, \sigma S(k, n)} \int_{\Lambda^{n}} d(x)_{n} \chi_{e}\left((x)_{n}\right) \mathbb{R}\left(\sigma^{\prime}(x)_{n}, P \sigma(x)_{n}\right),
\end{aligned}
$$

and so by Lemma IV.3.2 we have

$$
\bar{F}_{q}^{k}\left(\left(\Delta_{1}, l_{1}\right), \ldots,\left(\Delta_{k}, l_{k}\right) \leqq k ! C_{1,2} \operatorname{Tr}_{\mathscr{H}_{n}(\Lambda)}\left(\chi_{e} P_{1} \mathbb{R} P_{1} P_{2} \chi_{e}\right) .\right.
$$

Thus if $P_{2}$ and $\chi_{e}$ commute with each other and if $\mathbb{K}$ is positive, one may use (3.3.4) to eliminate $P_{2}$ from the above trace. Then our job will be done. Although $P_{2}$ does not commute with $\chi_{e}$, we will show that $P_{2}$ and $\chi_{e}$ almost commute with 
each other. To do this we use (4.3.20) to obtain

$$
\begin{aligned}
\operatorname{Tr}_{\mathscr{H}_{n}}\left(\chi_{e} P_{1} \mathbb{K} P_{1} P_{2} \chi_{e}\right)= & \sum_{\sigma^{\prime \prime} \in S(k, n)} \operatorname{Tr}_{\mathscr{H}_{n}}\left(\chi_{e} P_{1} \mathbb{K} P_{1} \chi_{\sigma^{\prime \prime}} P_{2} \chi_{e}\right), \\
\operatorname{Tr}_{\mathscr{H}_{n}}\left(\chi_{e} P_{1} \mathbb{K} P_{1} \chi_{\sigma^{\prime \prime}} P_{2} \chi_{e}\right)= & C_{1,2}^{-1} \sum_{P \in \tilde{E}_{k}\left(l_{1}, \ldots, l_{k}\right)} \sum_{\sigma^{\prime}, \sigma \in S(k, n)} \\
& \cdot \int_{\Lambda^{n}} d(x)_{n} \chi_{e}\left((x)_{n}\right) \mathbb{K}\left(\sigma^{\prime}(x)_{n}, P \sigma(x)_{n}\right) \chi_{e}\left(P \sigma^{\prime \prime}(x)_{n}\right) .
\end{aligned}
$$

We define

$$
\begin{aligned}
A(k, n) & =\left\{\sigma^{\prime \prime} \in S(k, n): \operatorname{Tr}_{\mathscr{H}}\left(\chi_{e} P_{1} \mathbb{K} P_{1} \chi_{\sigma^{\prime \prime}} P_{2} \chi_{e}\right) \neq 0\right\}, \\
\gamma & =\max \left\{l_{1}, \ldots, l_{k}\right\}, M_{\gamma}=\left[2\left|\Lambda_{q+\gamma+2}\right|^{1+(l-1) / p}\right] .
\end{aligned}
$$

We then have the following:

\section{Lemma IV.3.2.}

$$
\operatorname{card}(A(k, n)) \leqq \operatorname{card}\left(\tilde{E}_{k}\left(l_{1}, \ldots, l_{k}\right)\right) M_{\gamma} ! /\left(M_{\gamma}-k\right) !
$$

Proof. Because of $\chi_{e}\left((x)_{n}\right) \chi_{e}\left(P \sigma^{\prime \prime}(x)_{n}\right)$ in the integrand in (4.3.25), the trace for $\sigma^{\prime \prime} \in S(k, n)$ will vanish if the following set

$$
\left\{(x)_{n}:\left\|x_{k+1}\right\| \leqq \cdots \leqq\left\|x_{n}\right\|\right\} \cap\left\{(x)_{n}:\left\|x_{P \sigma^{\prime \prime}(k+1)}\right\| \leqq \cdots \leqq\left\|x_{P \sigma^{\prime \prime}(n)}\right\|\right\}
$$

has measure zero for any $P \in \widetilde{E}_{k}\left(l_{1}, \ldots, l_{k}\right)$. This means that for given $\sigma^{\prime \prime}$ the sequence $\left\langle P \sigma^{\prime \prime}(m): m \geqq k+1\right.$ and $\left.P \sigma^{\prime \prime}(m) \geqq k+1\right\rangle$ must be a subsequence of $\langle k+1, k+2, \ldots, n\rangle$ for some $P$ to give non-zero contributions. Since $\sigma^{\prime \prime} \in S(k, n)$, we have $P \sigma^{\prime \prime}(i)=P(i), i \leqq k$, for any $\sigma^{\prime \prime} \in S(k, n)$. We assert that if

$$
P \sigma^{\prime \prime}(m) \leqq k \text { for some } m>M_{\gamma},
$$

then the integrals in (4.3.25) are zero. We note that $x_{i} \in \Lambda_{q+\gamma+2}$ for $i \leqq k\left(\omega_{i} \in \mathscr{E}_{\Delta_{t}, l_{l}}^{(i)}\right)$. Thus, if (4.3.27) holds, $M_{\gamma}$-integration variables in (4.3.25) must be restricted to $\Lambda_{q+\gamma+2}$ by the factor $\chi_{e}\left(P \sigma^{\prime \prime}(x)_{n}\right)$. This is impossible by (4.1.14) and the definition of $\mathbb{K}\left((x)_{n},(y)_{n}\right)$. This proves our assertion. For given $P \in \widetilde{E}_{k}\left(l_{1}, \ldots, l_{k}\right), P \sigma^{\prime \prime} \equiv \pi$ will have the following form.

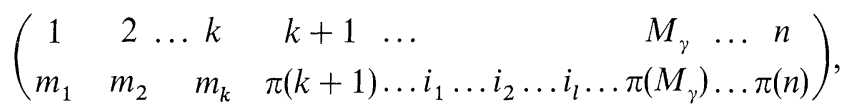

where $i_{j} \in\{1,2, \ldots, k\}$ and $m_{i}=P(i) \leqq M_{\gamma}$. By the assertion, $m \leqq M_{\gamma}$ for $\pi(m)=i_{j}$. We recall that the sequence $\left\langle\pi(m): m \geqq k+1\right.$ and $\left.\pi(m) \neq i_{l}\right\rangle$ in (4.3.28) must be a subsequence of $\langle 1,2, \ldots, n\rangle$, and so $\pi\left(M_{\gamma}+1\right)=M_{\gamma}+1, \ldots, \pi(n)=n$ in (4.3.28). For fixed $P$, the $i_{j}$ 's will be moved if one changes $\sigma^{\prime \prime} . \sigma^{\prime \prime}$ should not change the order of $\pi(m)$. Thus, for given $P$ there will be at most $k !\left(\begin{array}{c}M_{\gamma} \\ k\end{array}\right)$ number of $\sigma^{\prime \prime}$ which belongs to $A(k, n)$. Varying $P \in \widetilde{E}_{k}\left(l_{1}, \ldots, l_{k}\right)$ we arrive at the conclusion in the lemma.

From (4.3.23), (4.3.24) and from the definition of $A(k, n)$ in (4.3.26), it follows that

$$
\bar{F}_{q}^{k}\left(\left(\Delta_{1}, l_{1}\right), \ldots,\left(\Delta_{k}, l_{k}\right)\right) \leqq k ! C_{1,2 \sigma^{\prime \prime}} \sum_{\substack{\sigma^{\prime \prime} . \in A(k, n) \\ \text { Tre }}} \operatorname{Tr}_{\mathscr{H}_{n}}\left(\chi_{e} P_{1} \mathbb{K} P_{1} \chi_{\sigma^{\prime \prime}} P_{2} \chi_{e}\right)
$$


The next step is to get a bound for (4.3.29). From (4.3.8) the following is obvious:

$$
\overline{\mathscr{E}}_{q}^{k}\left(\left(\Delta_{1}, l_{1}\right), \ldots,\left(\Delta_{k}, l_{k}\right)\right) \subset\left(\begin{array}{cc}
\stackrel{\mathscr{C}}{x}_{i=1}^{(i)} & \mathscr{E}_{\Delta_{i}, l_{i}}^{(i)}
\end{array}\right) \times \Omega^{n-k} .
$$

From the definition of $\mathbb{K}\left((x)_{n},(y)_{n}\right)$ in (4.3.11) it follows that

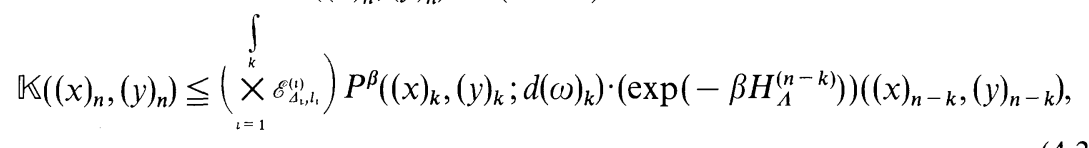

where we have used the fact that

$$
\exp \left(-\beta H_{A}^{(n-k)}\right)\left((x)_{n-k},(y)_{n-k}\right)=\int_{\Omega^{n-k}} P_{A}^{\beta}\left((x)_{n-k},(y)_{n-k} ; d(\omega)_{n-k}\right) \exp \left(-U\left((\omega)_{n-k}\right)\right) .
$$

We now use the definition of $\mathbb{K}_{\Delta, l}(x, y)$ and $\overline{\mathbb{K}}_{\Delta, l}(x, y)$ in (3.2.5) and (3.2.7) respectively into (4.3.30), and then use Proposition III.2.2 to arrive at

$$
\mathbb{K}\left((x)_{n},(y)_{n}\right) \leqq \prod_{i=1}^{k}\left[c_{1} \exp \left(-\frac{l_{i}^{2}}{16 \beta}\right)\right] Y\left((x)_{n},(y)_{n}\right),
$$

where

$$
Y\left((x)_{n},(y)_{n}\right)=\left[\prod_{i=1}^{k} \overline{\mathbb{K}}_{\Delta_{i}, l_{i}}\left(x_{i}, y_{i}\right)\right]\left[\exp \left(-\beta H_{A}^{(n-k)}\right)\right]\left((x)_{n-k},(y)_{n-k}\right)
$$

Notice that the above is the kernel of the operator

$$
Y \equiv\left(\prod_{i=1}^{k} \overline{\mathbb{K}}_{\Delta_{i}, l_{i}}\right) \exp \left[-\beta H_{A}^{(n-k)}\right]
$$

which is a positive operator on $\mathscr{H}_{n}\left(\mathbb{R}^{v}\right)$. From (4.3.25), (4.3.29) and (4.3.32), it follows that

$$
\begin{aligned}
& \bar{F}_{q}^{k}\left(\left(\Delta_{1}, l_{1}\right), \ldots,\left(\Delta_{k}, l_{k}\right)\right) \\
& \quad \leqq k ! C_{1,2}\left[\prod_{i=1}^{k} c_{1} \exp \left(-\frac{l_{i}^{2}}{16 \beta}\right)\right] \sum_{\sigma^{\prime \prime}: \in A(k, n)} \operatorname{Tr}_{\mathscr{H}_{n}}\left(\chi_{e} P_{1} Y P_{1} \chi_{\sigma^{\prime \prime}}: P_{2}\right) .
\end{aligned}
$$

We now use the positivity of $Y$ and the abstract Hölder's inequality [11] to conclude that

$$
\begin{aligned}
\operatorname{Tr}_{\mathscr{H}_{n}}\left(\chi_{e} P_{1} Y P_{1} \chi_{\sigma^{\prime \prime}} P_{2}\right) & \leqq\left[\operatorname{Tr}_{\mathscr{H}_{n}}\left(\chi_{e} P_{1} Y P_{1}\right)\right]^{1 / 2}\left[\operatorname{Tr}_{\mathscr{H}_{n}}\left(P_{2} \chi_{\sigma^{\prime \prime}} P_{1} Y P_{1} \chi_{\sigma^{\prime \prime}} P_{2}\right)\right]^{1 / 2} \\
& \leqq\left[\operatorname{Tr}_{\mathscr{H}_{n}}\left(\chi_{e} P_{1} Y P_{1}\right)\right]^{1 / 2}\left[\operatorname{Tr}_{\mathscr{H}_{n}}\left(\chi_{\sigma^{\prime \prime}} P_{1} Y P_{1}\right)\right]^{1 / 2}
\end{aligned}
$$

A direct computation gives us that for any $\sigma^{\prime \prime} \in S(k, n)$,

$$
\begin{aligned}
\operatorname{Tr}_{\mathscr{H}_{n}}\left(\chi_{\sigma^{\prime \prime}} P_{1} Y P_{1}\right) & =\operatorname{card}\left(S^{\prime}(k, n)\right)^{-2} \sum_{\sigma^{\prime}, \sigma \in S(k, n)} \int d(x)_{n} \chi_{e}\left(\sigma^{\prime \prime}(x)_{n}\right) Y\left(\sigma^{\prime}(x)_{n}, \sigma(x)_{n}\right) \\
& =[(n-k) !]^{-2} \sum_{\sigma^{\prime}, \sigma \in S(k, n)} \int d(x)_{n} \chi_{e}\left(\sigma^{\prime}(x)\right) Y\left((x)_{n}, \sigma(x)_{n}\right)
\end{aligned}
$$




$$
\begin{aligned}
& =[(n-k) !]^{-2} \sum_{\sigma \in S(k, n)} \int d(x)_{n} Y\left((x)_{n}, \sigma(x)_{n}\right) \\
& =[(n-k) !]^{-1} \operatorname{Tr}_{\mathscr{H}_{n}}\left(P_{1} Y P_{1}\right) .
\end{aligned}
$$

We substitute the above equality into (4.3.36), and then we use the definitions of $P_{1}$ and $Y$ in (4.3.21) and (4.3.34) respectively to obtain

$$
\begin{aligned}
& \operatorname{Tr}_{\mathscr{H}_{n}}\left(\chi_{e} P_{1} Y P_{1} \chi_{\sigma^{\prime \prime}} P_{2}\right) \leqq \frac{1}{(n-k) !} \operatorname{Tr}_{\mathscr{H}_{n}}\left(P_{1} Y P_{1}\right) \\
& =\frac{1}{(n-k) !}\left[\prod_{i=1}^{k} \operatorname{Tr}_{L^{2}(\Lambda)}\left(\overline{\mathbb{K}}_{\Delta_{i}, l_{i}}\right)\right] \operatorname{Tr}_{\mathscr{H}_{n-k}^{(s)}}^{\left(\exp \left[-\beta H_{\Lambda}^{(n-k)}\right]\right)} \\
& \leqq \frac{1}{(n-k) !} c_{2}^{k} \prod_{i=1}^{k}\left(2 l_{i}+2\right)^{v} \operatorname{Tr}_{\mathscr{H}_{n}^{(s)} k}\left(\exp \left[-\beta H_{\Lambda}^{(n-k)}\right]\right)
\end{aligned}
$$

for any $\sigma^{\prime \prime} \in S^{\prime}(k, n)$. Here we have used Proposition III.2.2 to obtain the third inequality in the above. We now combine (4.3.35) and (4.3.37) to obtain

$$
\bar{F}_{q}^{k}\left(\left(\Delta_{1}, l_{1}\right), \ldots,\left(\Delta_{k}, l_{k}\right)\right) \leqq M\left(k, q ; l_{1}, \ldots, l_{k}\right) \operatorname{Tr}_{\mathscr{H}_{n}^{(s)} k}\left(\exp \left[-\beta H_{A}^{(n-k)}\right]\right)
$$

where

$$
M\left(k, q ; l_{1}, \ldots, l_{k}\right)=\operatorname{card}(A(k, n)) k ! C_{1,2} \frac{1}{(n-k) !} c^{k} \prod_{i=1}^{k}\left(2 l_{i}+2\right)^{v} \exp \left(-\frac{l_{i}^{2}}{16 \beta}\right) .
$$

From the bound in Lemma IV.3.2,

$$
\operatorname{card}(A(k, n)) \leqq \operatorname{card}\left(\tilde{E}_{k}\left(l_{1}, \ldots, l_{k}\right) M_{\gamma}^{k},\right.
$$

and from the definition of $M_{\gamma}$ in (4.3.26) it is easy to check that there are constants $c^{\prime}$ and $c^{\prime \prime}$ such that

$$
\begin{aligned}
M_{\gamma}^{k} & \leqq \exp \left(c^{\prime} k \log \left|\Lambda_{q+1}\right|+c^{\prime} k \log (\gamma+2)\right) \\
& \leqq \exp \left(c^{\prime \prime} k \log \left|\Lambda_{q+1}\right|+c^{\prime \prime}(\gamma+2)\right) .
\end{aligned}
$$

Here we have used the fact that $k \log x<2 x+k \log k$ for $x \geqq 1$, and the bound in (4.1.13). From (4.3.16) and (4.3.12) we also have that for some $d_{1}$,

$$
\operatorname{card}\left(\tilde{E}_{k}\left(l_{1}, \ldots, l_{k}\right)\right) \leqq \exp \left[d_{1} k \log \left|\Lambda_{q+1}\right|\right] \prod_{i=1}^{k} \exp \left(d_{1}\left(l_{i}+2\right)\right)
$$

We recall that $\gamma=\max \left\{l_{i}\right\}$. We combine the definition of $C_{1,2}$ in (4.3.21), and (4.3.40) and (4.3.41) to (4.3.39) to conclude that there are constants $c_{1}$ and $c_{2}$ such that

$$
M\left(k, q ; l_{1}, \ldots, l_{k}\right) \leqq c_{1}^{k} \exp \left(c_{2} k \log \left|\Lambda_{q+1}\right|\right)(n-k) ! \prod_{i=1}^{k} \exp \left[-\frac{l_{i}^{2}}{32 \beta}\right]
$$

We now substitute (4.3.42) into (4.3.38), and then (4.3.38) into (4.3.10) to obtain 
the following bound:

$$
\begin{aligned}
\bar{F}_{q, k}^{(n)} \leqq & c_{1}^{k} \exp \left(c_{2} k \log \left|\Lambda_{q+1}\right|\right) \operatorname{Tr}_{\mathscr{H}_{n-k}}^{(s)}\left(\exp \left(-\beta H_{\Lambda}^{(n-k)}\right)\right. \\
& \cdot \prod_{i=1}^{k}\left[\sum_{\Lambda_{\imath} \subset \Lambda_{q}} \sum_{l_{l}=-1}^{\infty} \exp \left[-\frac{1}{32 \beta} l_{i}^{2}+4 v b\left(l_{i}+2\right)^{2}\right]\right. \\
\leqq & c_{1}^{k} \exp \left(2 c_{2} k \log \left|\Lambda_{q+1}\right|\right) \operatorname{Tr}_{\mathscr{H}_{n}^{(s)} k}\left(\exp \left(-\beta H_{A}^{(n-k)}\right)\right) .
\end{aligned}
$$

Here we have chosen $b$ such that $4 v b<1 / 32 \beta$ to get the second inequality. Using (4.1.13) and the fact that $\gamma\left(v, p^{\prime}\right)>1$, it follows that for any constant $|\alpha|, c_{1}, c_{2}$ and $c$ there are constants $D_{1}, D_{2}, D_{3}>0$ such that

$$
\begin{aligned}
c_{1} k & +2 c_{2} k \log \left|\Lambda_{q+1}\right|+|\alpha|\left|\Lambda_{q+1}\right|^{1+(l-1) / p}-c\left|\Lambda_{q+1}\right|^{1+(l-1) / p \gamma\left(v, p^{\prime}\right)} \\
& \leqq D_{1}-D_{2} q-D_{3} k
\end{aligned}
$$

by (4.1.13). We finally combine (4.3.43), (4.3.1) and (4.1.19), and we then use (4.3.44) to obtain

$$
G_{q, k}^{(n)} \leqq \Xi_{\Lambda}^{-1} z^{n} \exp \left(D_{1}-D_{2} q-D_{3} k\right) \operatorname{Tr}_{\mathscr{H}_{n}(s) k}\left(\exp \left(-\beta H_{\Lambda}^{(n-k)}\right)\right)
$$

for some positive constants $D_{1}, D_{2}$ and $D_{3}$. Thus

$$
\sum_{n=0}^{\infty} \sum_{q \geqq p_{0}} \sum_{k \geqq\left|\Lambda_{q}\right| l / p}^{n} G_{q, k}^{(n)} \leqq e^{D} \Xi_{\Lambda}^{-1}\left(\sum_{n^{\prime}=0}^{\infty} z^{n^{\prime}} \operatorname{Tr}_{\mathscr{H}_{n^{\prime}}^{(s)}}\left(-\beta H_{\Lambda}^{\left(n^{\prime}\right)}\right)\right)=e^{D}
$$

for some $D>0$. The theorem now follows from (4.1.15), (4.1.18) and the above bound. This completes the proof of the main theorem.

\section{IV.4. The Proof of Theorem IV.2.1.}

In this section we produce the proof of Theorem IV.2.1, and so we complete the proof of Theorem II.3.1. From (4.2.5) we recall that for any configuration $(\omega)_{n}$ in $\overline{\mathscr{E}}_{q, k}$ there is $\tau_{0} \in[0, \beta]$ such that

$$
\sum_{r \in \Lambda_{q}} \bar{n}\left(r, \tau_{0}\right)^{p} \geqq\left|\Lambda_{q}\right|^{l}
$$

where $\bar{n}\left(r, \tau_{0}\right)$ is the number of $\omega_{i}, i=1,2, \ldots, k$, such that $\omega_{i}\left(\tau_{0}\right) \in Q(r)$. If the interaction satisfies either Assumption $A$ (strong superstability) or else Assumption $A^{\prime}$ (positivity) in Sect. II.2, we have

$$
U_{1}\left((\omega(\tau))_{k} \geqq \sum_{r \in \Lambda}\left(A \bar{n}(r, \tau)^{p^{\prime}}-B \bar{n}(r, \tau)\right],\right.
$$

where $U_{1}\left((\omega(\tau))_{k}\right)$ has been defined in (4.2.3). From the definition of $\overline{\mathscr{E}}_{q, k}$ in (4.2.1) we also have that for any $q^{\prime}>q$,

$$
\sum_{r \in \Lambda_{q}} n(r, \tau)^{p}<\left|\Lambda_{q}^{\prime}\right|^{l} \quad \text { on } \overline{\mathscr{E}}_{q, k} .
$$

For a given configuration of paths $(\omega)_{n}$ in $\overline{\mathscr{E}}_{q, n}$, we pick $\tau_{0} \in[0, \beta]$ so that (4.4.1) is 
satisfied, and we define

$$
V\left(\left(\left(\omega(\tau)_{k}\right) \equiv \frac{1}{\beta} \sum_{i=1}^{k}\left|\omega_{i}(\tau)-\omega_{i}\left(\tau_{0}\right)\right|^{2}\right.\right.
$$

From the definition of $V\left((\omega)_{k}\right)$ in (4.2.8) it follows that

$$
V\left((\omega)_{k}\right) \geqq \int_{0}^{\beta} V\left((\omega(\tau))_{k}\right) d \tau .
$$

For $p^{\prime} \geqq 2$ we have written in (4.2.9)

$$
\gamma\left(v, p^{\prime}\right)=1+\frac{2\left(p^{\prime}-1\right)}{v p^{\prime}+(2-v)} .
$$

If the interaction satisfies Assumption $A$, the following inequality holds:

$$
\frac{p^{\prime}}{p^{\prime}-1}<\gamma\left(v, p^{\prime}\right)
$$

For the interactions satisfying Assumption $A$ we choose $p>l>1$ such that the following inequalities hold:

$$
\begin{gathered}
l / p<2 / v \\
\frac{p^{\prime}}{p^{\prime}-1}<p<\gamma\left(v, p^{\prime}\right) .
\end{gathered}
$$

In Lemma IV.2.2. we have shown that, if $v \leqq 3$, we can choose $p>l>1$ so that (4.4.8) and (4.4.9) holds simultaneously. We will discuss other choices of $p$ and $l$ in the next section in more detail. For the replusive interaction (Assumption $A^{\prime}$ ) we only impose the condition

$$
p<\gamma\left(v, p^{\prime}\right)
$$

We now begin to prove Theorem IV.2.1. For any fixed constant $a, b>0$ and for a given configuration of paths $(\omega)_{n}$ in $\overline{\mathscr{E}}_{q, k}$, we write

$$
B(\tau) \equiv a U_{1}\left(\left(\omega(\tau)_{k}\right)+b V\left((\omega(\tau))_{k}\right) .\right.
$$

We have the following:

Proposition IV.4.1. For $1<l<p$, there exist constants $c_{1}>0$ and $c_{2}>0$ such that

$$
B(\tau) \geqq c_{1} \sum_{r \in \Lambda_{q}} \bar{n}\left(r, \tau_{0}\right)^{\gamma\left(v, p^{\prime}\right)}-c_{2}\left|\Lambda_{q+1}\right|^{1+((l-1) / p)}
$$

for any given $(\omega)_{n}$ in $\overline{\mathscr{E}}_{q, k}$.

Proof. From (4.4.2) and (4.4.4) it follows that

$$
B(\tau) \geqq \sum_{r \in \Lambda}\left[a A \bar{n}(r, \tau)^{p^{\prime}}+\frac{b}{\beta} \sum_{\omega_{i}(\tau) \in Q(r)}\left|\omega_{i}(\tau)-\omega_{i}\left(\tau_{0}\right)\right|^{2}\right]-a B k .
$$

Let $\bar{n}_{r^{\prime}}(r, \tau)$ be the number of paths $\omega_{i}, i=1,2, \ldots, k$, such that $\omega_{i}(\tau) \in Q(r)$ and 
$\omega_{i}\left(\tau_{0}\right) \in Q\left(r^{\prime}\right)$. Since $\omega_{i}\left(\tau_{0}\right) \in \Lambda_{q}$ for any $i=1,2, \ldots, k$, we have

$$
\bar{n}(r, \tau)=\sum_{r^{\prime} \in \Lambda_{q}} \bar{n}_{r^{\prime}}(r, \tau)
$$

Since $\bar{n}(r, \tau)$ and $\bar{n}_{r^{\prime}}(r, \tau)$ are non-negative integers, we have that for any $p^{\prime} \geqq 2$ (see Lemma IV.2.2. below)

$$
\bar{n}(r, \tau)^{p^{\prime}} \geqq \sum_{r^{\prime} \in \Lambda_{q}} \bar{n}_{r^{\prime}}(r, \tau)^{p^{\prime}}
$$

Thus

$$
B(\tau) \geqq \sum_{r^{\prime} \in \Lambda_{q}}\left[a A \sum_{r \in \Lambda} \bar{n}_{r^{\prime}}(r, \tau)^{p}+\frac{b}{\beta} \sum_{\begin{array}{c}
\omega_{i}(\tau) \in Q(r): \\
\omega_{\imath}(\tau) \in Q\left(r^{\prime}\right)
\end{array}}\left|\omega_{i}(\tau)-\omega_{i}\left(\tau_{0}\right)\right|^{2}\right]-a B k
$$

As before we denote $[a]$ the greatest integer number which is less than or equal to $a$.

We first consider the case in which for a given $q>0$,

$$
\text { card }\left(\left\{\omega_{i}: \omega_{i}\left(\tau_{0}\right) \in Q\left(r^{\prime}\right) \text { and }\left|\omega_{i}(\tau)-\omega_{i}\left(\tau_{0}\right)\right| \leqq \bar{n}\left(r^{\prime}, \tau_{0}\right)^{q}\right\}\right) \geqq\left[\frac{1}{2} \bar{n}\left(r^{\prime}, \tau_{0}\right)\right] \text {. }
$$

Note that

$$
\sum_{r \in \Lambda} \bar{n}_{r^{\prime}}(r, \tau)^{p^{\prime}} \geqq \sum_{r:\left|r-r^{\prime}\right| \leqq n\left(r^{\prime}, \tau_{0}\right)^{q}} \bar{n}_{r^{\prime}}(r, \tau)^{p^{\prime}} .
$$

Since the number of $r$ 's such that $\left|r-r^{\prime}\right| \leqq \bar{n}\left(r^{\prime}, \tau_{0}\right)^{q}$ is not larger than $\left[2 \bar{n}\left(r^{\prime}, \tau_{0}\right)^{q}+1\right]^{v}$, we use Hölder's inequality (or Jensen's inequality) to obtain

$$
\begin{aligned}
\sum_{r \in \Lambda} \bar{n}_{r^{\prime}}(r, \tau)^{p^{\prime}} & \geqq\left[2 \bar{n}\left(r^{\prime}, \tau_{0}\right)^{q}+1\right]^{v}\left(\left[2 \bar{n}\left(r^{\prime}, \tau_{0}\right)^{q}+1\right]^{-v}\left[\frac{1}{2} \bar{n}\left(r^{\prime}, \tau_{0}\right)\right]\right)^{p^{\prime}} \\
& \geqq c_{1}^{\prime}\left(v, p^{\prime}\right) \bar{n}\left(r^{\prime}, \tau_{0}\right)^{v q} \bar{n}\left(r^{\prime}, \tau_{0}\right)^{(1-v q) p^{\prime}}-c_{2}^{\prime}(v, p)
\end{aligned}
$$

for some constant $c_{1}^{\prime}, c_{2}^{\prime}>0$. On the other hand if

$$
\operatorname{card}\left(\left\{\omega_{i}: \omega_{i}\left(\tau_{0}\right) \in Q\left(r^{\prime}\right) \text { and }\left|\omega_{i}(\tau)-\omega_{i}\left(\tau_{0}\right)\right| \leqq \bar{n}\left(r^{\prime}, \tau_{0}\right)^{q}\right\}\right)<\left[\frac{1}{2} \bar{n}\left(r^{\prime}, \tau_{0}\right)\right]
$$

then

$$
\begin{aligned}
\sum_{\substack{\omega_{i} \in Q(r): \\
\omega_{i}\left(\tau_{0}\right) \in Q\left(r^{\prime}\right)}}\left|\omega_{i}(\tau)-\omega_{i}\left(\tau_{0}\right)\right|^{2} & \geqq\left[\frac{1}{2} \bar{n}\left(r^{\prime}, \tau_{0}\right)\right]\left(n\left(r^{\prime}, \tau_{0}\right)^{q}-1\right)^{2} \\
& \geqq c_{1}^{\prime} \bar{n}\left(r^{\prime}, \tau_{0}\right)^{1+2 q}-c_{2}^{\prime}
\end{aligned}
$$

for some constant $c_{1}^{\prime}, c_{2}^{\prime}>0$. By choosing $q$ such that

$$
v q+(1-v q) p^{\prime}=1+2 q
$$

(i.e., $q=\left(p^{\prime}-1\right) /\left[v p^{\prime}+(2-v)\right]$, and by (4.1.13) we have proven the proposition.

The following facts are probably well known. But we produce the proof.

Lemma IV.4.2. Let $\{n(1), n(2), \ldots, n(k)\}$ be a finite sequence of non-negative real 
numbers. Then
(a) $\sum_{i=1}^{k} n(i)^{p} \leqq\left(\sum_{i=1}^{k} n(i)\right)^{p}, \quad p \geqq 1$,
(b) $\sum_{i=1}^{k} n(i)^{q} \geqq\left(\sum_{i=1}^{k} n(i)\right)^{q}, \quad 0 \leqq q \leqq 1$.

Proof. (a) The inequality obviously holds if $p$, is a natural number. For a non-natural number $p$, the inequality follows from the Hadamard three line theorem [11] for the function

$$
f(z)=\sum_{i=1}^{k} n(i)^{p+z}
$$

with a non-negative integer $p$.

(b) The inequality holds for $q=0$ and $q=1$. For $M>0$ and $0<q<1$, let $f$ be the function on $\left[0, \frac{1}{2} M\right]$ defined by $f(x)=(M-x)^{q}+x^{q}$. Then it is easy to check that $f(x)$ is an increasing function on $\left[0, \frac{1}{2} M\right]$, thus if we set $n(1)=M-x$ and $n(2)=x$, then $n(1)+n(2)=M$ and $n(1)^{q}+n(2)^{q}$ is increasing as $|n(1)-n(2)|$ tends to zero. Now the lemma follows from an induction argument.

Theorem IV.4.3. Let $1<l<p$ be chosen such that $p \leqq \gamma\left(v, p^{\prime}\right)$. Then for any given $a>0$ and $b>0$ there exist constants $c_{1}>0$ and $c_{2}>0$ such that

$$
a U_{1}\left((\omega)_{k}\right)+b V\left((\omega)_{k}\right) \geqq c_{1}\left|\Lambda_{q}\right|^{1+\left((l-1) \gamma\left(v, p^{\prime}\right) / p\right)}-c_{2}\left|\Lambda_{q+1}\right|^{1+(l-1) / p)}
$$

on $\overline{\mathscr{E}}_{q, k}$.

Proof. We note that from (4.4.5) and (4.4.11)

$$
a U_{1}\left((\omega)_{k}\right)+b V\left((\omega)_{k}\right) \geqq \int_{0}^{\beta} B(\tau) d \tau .
$$

Let $p$ and $\gamma$ be given numbers. If $1 \leqq p \leqq \gamma$,we use Hölder's inequality to obtain

$$
K^{-1} \sum_{i=1}^{k} n(i)^{p} \leqq\left(K^{-1} \sum_{i=1}^{k} n(i)^{\gamma}\right)^{p / \gamma}, 1 \leqq p \leqq \gamma .
$$

If $1 \leqq \gamma \leqq p$, one may use Lemma IV.4.2. (b) to obtain

$$
\sum_{i=1}^{k} n(i)^{\gamma} \geqq\left[\sum_{i=1} n(i)^{p}\right]^{\gamma / p}, \quad 1 \leqq \gamma \leqq p .
$$

The above inequalities imply that

$$
\begin{aligned}
& \sum_{r \in \Lambda_{q}} \bar{n}\left(r, \tau_{0}\right)^{\gamma} \geqq\left|\Lambda_{q}\right|^{1+(l-1) \gamma / p}, \quad \gamma \geqq p, \\
& \sum_{r \in \Lambda_{q}} \bar{n}\left(r, \tau_{0}\right)^{\gamma} \geqq\left|{ }^{\Lambda}{ }_{q}\right|^{l \gamma / p}, \quad \gamma \leqq p .
\end{aligned}
$$

If one chooses $\gamma=\gamma\left(v, p^{\prime}\right)$, then the theorem follows (4.4.13), Proposition IV.4.1. and (4.4.12) 
Proof of Theorem IV.2.1 (b). The theorem is a consequence of Theorem IV.4.3. and the fact that $\gamma\left(v, p^{\prime}\right)>1$ for any $v$ and $p^{\prime} \geqq 2$.

The rest of this section is devoted to prove Theorem IV.2.1. (a). We write $\mathbb{K}(\omega) \equiv\left\{\omega_{1}{ }^{\prime} \omega_{2}, \ldots, \omega_{k}\right\}$. Applying Assumption $A\left(\right.$ b) in Sect. II.2, we have that on $\overline{\mathscr{E}}_{q, k}$

$$
\begin{aligned}
W\left((\omega)_{k},(\omega)_{n-k}\right) & =\frac{1}{2} \sum_{\substack{\left.\omega_{j} \in \mathbb{R}(\omega) \\
\omega j \in \mathbb{K}(\omega)\right)^{c}}} \int_{o}^{\beta} \Phi\left(\omega_{i}(\tau)-\omega_{j}(\tau)\right) d \tau \\
& \geqq-\frac{1}{2} \sum_{\substack{\omega_{i} \in \mathbb{K}(\omega) \\
\omega_{j} \in \mathbb{K}(\omega)^{c}}}^{\beta} \int_{0}^{\beta} \varphi\left(\left|\omega_{i}(\tau)-\omega_{j}(\tau)\right| d \tau\right. \\
& \geqq-c \int_{0}^{\beta} \sum_{\substack{r \in A \\
r^{\prime} \in A}} \varphi\left(\left|r-r^{\prime}\right|\right) n(r, \tau) \bar{n}\left(r^{\prime}, \tau\right) d \tau
\end{aligned}
$$

for some constant $c$, where $\bar{n}\left(r^{\prime}, \tau\right)=$ card $\left(\left\{\omega_{i}(\tau): \omega_{i} \in \mathbb{K}(\omega)\right.\right.$ and $\left.\left.\omega_{i}(\tau) \in Q\left(r^{\prime}\right)\right\}\right)$. For a given configuration of paths $(\omega)_{n}$ in $\overline{\mathscr{E}}_{q, k}$ we write.

$$
\begin{aligned}
& W_{1}(\tau) \equiv c \sum_{\substack{r \in A_{4 q} \\
r \in A_{2 q}}}\left(\left|r-r^{\prime}\right|\right) n(r, \tau) \bar{n}\left(r^{\prime}, \tau\right), \\
& W_{2}(\tau) \equiv c \sum_{\substack{r \in \mathcal{A}_{q} \\
r^{\prime} \in \in_{2 q}}} \varphi\left(\left|r-r^{\prime}\right|\right) n(r, \tau) \bar{n}\left(r^{\prime}, \tau\right), \\
& W_{3}(\tau) \equiv c \sum_{\substack{r \in \Lambda_{\mathcal{G}}^{\mathrm{q}} \\
r \in \Lambda_{2 q}}} \varphi\left(\left|r-r^{\prime}\right|\right) n(r, \tau) \bar{n}\left(r^{\prime}, \tau\right) .
\end{aligned}
$$

From (4.4.15) it follows that

$$
\left.W(\omega)_{k},(\omega)_{n-k}\right) \geqq-\int_{0}^{\beta}\left[W_{1}(\tau)+W_{2}(\tau)+W_{3}(\tau)\right] d \tau .
$$

For any given constant $a>0$, we use (4.4.2) to obtain that on $\overline{\mathscr{E}}_{q, k}$,

$$
\begin{aligned}
a U_{1}\left((\omega(\tau))_{k}\right)-W_{1}(\tau) & \geqq a A \sum_{r^{\prime} \in \Lambda_{2 q}} \bar{n}\left(r^{\prime}, \tau\right)^{p^{\prime}}-c \sum_{\substack{r \in A_{4 q} \\
r^{\prime} \in \Lambda_{2 q}}} \varphi\left(\left|r-r^{\prime}\right| \bar{n}\left(r^{\prime}, \tau\right) n(r, \tau) n(r, \tau)-a B k\right. \\
& \geqq \sum_{\substack{r \in A_{4 q} \\
r^{\prime} \in \Lambda_{2 q}}} \varphi\left(\left|r-r^{\prime}\right|\right)\left[a^{\prime} \bar{n}\left(r^{\prime}, \tau\right)^{p^{\prime}}-c \bar{n}\left(r^{\prime}, \tau\right) n(r, \tau)\right]-a B k,
\end{aligned}
$$

where $a^{\prime}=a A\left[\sum_{r \in Z^{\prime}} \varphi(|r|)\right]^{-1}$. We note that $a^{\prime}|x|^{p^{\prime}}-c|x||y| \geqq-c^{\prime}|y|^{p^{\prime} / p^{\prime}-1}$ for some constant $c^{\prime}$, and so

$$
\begin{aligned}
a U_{1}\left((\omega(\tau))_{k}\right)-W_{1}(\tau) & \geqq-c^{\prime} \sum_{\substack{r \in A_{4 q} \\
r^{\prime} \in \Lambda_{2 q}}} \varphi\left(\left|r-\tau^{\prime}\right|\right) n(r, \tau)^{p^{\prime} \mid p^{\prime}-1}-c_{1}^{\prime \prime} k \\
& \geqq-c_{1}^{\prime} \sum_{r \in \Lambda_{4 q}} n(r, \tau)^{p^{\prime} / p^{\prime}-1}-c_{1}^{\prime \prime} k \\
& \geqq-c_{1}^{\prime}\left|\Lambda_{4}{ }^{q}\right|\left[\left|\Lambda_{4 q}\right|^{-1} \sum_{r \in \Lambda_{4 q}} n(r, \tau)^{p}\right]^{p^{\prime} / p\left(p^{\prime}-1\right)}-c_{1}^{\prime \prime} \\
& \geqq-c_{1}\left|\Lambda_{4 q}\right|^{1+\left((l-1) p^{\prime} / p\left(p^{\prime}-1\right)\right.}-c_{1}^{\prime \prime} k
\end{aligned}
$$


Here we have used the condition (4.4.9), Hölder's inequality and (4.4.3) to derive the above inequality. If one choose $p \leqq p^{\prime} / p^{\prime}-1$, one may use Lemma IV. 4.2(a) instead of Hölder's inequality to obtain the bound:

$$
a U\left((\omega(\tau))_{k}\right)-W_{1}(\tau) \geqq-c_{1}^{\prime}\left|\Lambda_{4 q}\right|^{l p^{\prime} / p\left(p^{\prime}-1\right)}-c_{1}^{\prime \prime} k .
$$

To estimate $W_{2}(\tau)$, we use (2.2.4), (4.4.8) and the fact that $n(r, \tau)<\left|\Lambda_{4 r}\right|^{l / p}<c|r|^{v l / p}$ for any $|r| \geqq q$ (by (4.4.3) and Lemma IV.4.2. (a)) to obtain

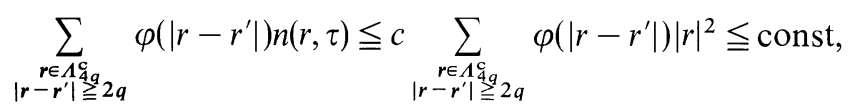

and so it follows from the definition of $W_{2}(\tau)$ in (4.4.16) that

$$
-W_{2}(\tau) \geqq-c_{2}^{\prime \prime} k
$$

for some constant $c_{2}^{\prime \prime}>0$. Finally we estimate $W_{3}(\tau)$. Since $\omega_{i}\left(\tau_{0}\right) \in \Lambda_{q}{ }^{\prime} i=1,2, \ldots, k$, we have

$$
\left|\omega_{i}(\tau)-\omega_{i}\left(\tau_{0}\right)\right|^{2} \geqq\left(\left|r^{\prime}\right|-q-1\right)^{2} \text { if } \omega_{i}(\tau) \in Q\left(r^{\prime}\right) \subset \Lambda_{2 q}^{c^{\prime}},
$$

and since $n(r, \tau)<|r|^{v / p}$ for $|r|>q$ by (4.4.3), it follows from (4.4.4) and (2.2.4) to conclude that for any given $b>0$

$$
\begin{aligned}
-W_{3}(\tau)+b V\left((\omega(\tau))_{k}\right) & \geqq-\sum_{r^{\prime} \in \Lambda_{2 q}^{\mathrm{c}}} \bar{n}\left(r^{\prime}, \tau\right)\left[c \sum_{r \in \Lambda_{4 q}^{\mathrm{c}}} \varphi\left(\left|r-r^{\prime}\right|\right)|r|^{v l / p}-\frac{b}{\beta}\left(\left|r^{\prime}\right|-q-1\right)^{2}\right] \\
& \geqq-\sum_{r^{\prime} \in \Lambda_{2 q}^{\mathrm{c}}} \bar{n}\left(r^{\prime}, \tau\right)\left[c^{\prime \prime}\left|r^{\prime}\right|^{v l / p}-\frac{b}{4 \beta}\left|r^{\prime}\right|^{2}+c^{\prime \prime \prime}\right] \\
& >0,
\end{aligned}
$$

if $p_{0}$ is sufficiently large $\left(q \geqq p_{0}\right)$. Since

$$
\begin{aligned}
\left|\Lambda_{4 q}\right| /\left|\Lambda_{q+1}\right| & \leqq \text { const } \\
k & \leqq\left|\Lambda_{q+1}\right| /\left.\right|^{1+(l-1) / p}
\end{aligned}
$$

by (4.1.13), integrating (4.4.18), (4.4.20) and (4.4.21) with respect to $\tau$ and using (4.4.17) and (4.4.5), we arrive at the following bound:

$$
W\left((\omega)_{k},(\omega)_{n-k}\right)+a U_{1}\left((\omega)_{k}+b V\left((\omega)_{k}\right) \geqq-c^{\prime \prime \prime}\left|\Lambda_{q+1}\right|^{1+\left((l-1) p^{\prime} / p\left(p^{\prime}-1\right)\right)}\right.
$$

We now use the assumption (4.4.7) and Theorem IV.4.3. to conclude that for any given $a>0, b>0$ there is a constant $c$ such that

$$
2 a U_{1}\left((\omega)_{k}\right)+2 b V\left((\omega)_{k}\right)+W\left((\omega)_{k},(\omega)_{n-k}\right) \geqq c\left|\Lambda_{q+1}\right|^{1+\left[(l-1) \gamma\left(v, p^{\prime}\right) / p\right]}
$$

on $\overline{\mathscr{E}}_{q, k}$. This completes the proof of Theorem IV.2.1. (a).

\section{Discussions}

There were two restrictions for choosing $1<l<p$ in the proof of Theorem 
IV.2.1. (a), namely, (4.4.8) and (4.4.9). We first discuss the condition (4.4.8):

$$
l / p<2 / v \text {. }
$$

We have used (5.1) to derive the bound in (4.4.21). That is, one has to bound the following:

$$
\left[a n(r, \tau)-b\left|\omega(\tau)-\omega\left(\tau_{0}\right)\right|^{2} \cong a|r|^{l v / p}-b|r|^{2} .\right.
$$

where $\omega(\tau) \in Q(r)$ and $\omega\left(\tau_{0}\right) \in \Lambda_{q}$, and $n(r, \tau)<\left|\Lambda_{|r|}\right|^{l / p}$ by (4.4.3). If (5.1) does not hold, more than $|r|^{2}$ - paths pass a unit cube $Q(r)$ at some time $\tau \in[0, \beta)$. In this case we are not be able to control $W_{3}(\tau)$ by the fluctuations of paths $V\left((\omega(\tau))_{k}\right)$. Thus the condition (5.1.) seems to be essential. If $v \geqq 4$, we have to choose $p \geqq 2$. Since $\gamma\left(v, p^{\prime}\right)$ $<1+(2 / v)$ for any $p^{\prime} \geqq 2, p>\gamma(v, p)$ for $v \geqq 4$. In this case we use (4.4.14) instead of (4.4.13) to obtain the result similar to that of Theorem IV.4.3. with the following replacement of $1+((l-1) / p) \gamma\left(v, p^{\prime}\right)$ by $(l / p) \gamma\left(v, p^{\prime}\right)$. One notices that $l \gamma\left(v, p^{\prime}\right) / p<1$ for $v \geqq 4$ if (5.1) holds. Thus we do not have enough convergent factors from the iteraction terms to control local number operators.

We next consider the condition (4.4.7):

$$
\frac{p^{\prime}}{p^{\prime}-1}<\gamma\left(v, p^{\prime}\right)
$$

The above condition gives the restriction on $p^{\prime}$ for the interaction satisfying Assumption A. If $\gamma\left(v, p^{\prime}\right)<p^{\prime} / p^{\prime}-1$, there are three possible ways to choose $p$. If we choose $p \leqq \gamma\left(v, p^{\prime}\right)$, the exponent in the right-hand side of (4.4.18) should be replaced by $l p^{\prime} / p\left(p^{\prime}-1\right)$ by Lemma IV.4.2 (b). Combining with Theorem IV.4.3 we need the following necessary condition to get the bound in Theorem IV.2.1:

$$
\frac{l}{p} \frac{p^{\prime}}{p^{\prime}-1}<1+\frac{l-1}{p} \gamma\left(v, p^{\prime}\right), \quad \text { for } p \leqq \gamma\left(v, p^{\prime}\right) \leqq \frac{p^{\prime}}{p^{\prime}-1} .
$$

Since $1-\gamma\left(v, p^{\prime}\right) / p \leqq 1$ if $p \leqq \gamma\left(v, p^{\prime}\right)$, the above is a contradiction. If we choose $\gamma\left(v, p^{\prime}\right) \leqq p \leqq p^{\prime} / p^{\prime}-1$, by the reason discussed in the previous paragraph, we have the following necessary condition:

$$
\frac{l}{p} \frac{p^{\prime}}{p^{\prime}-1}<\frac{l}{p} \gamma\left(v, p^{\prime}\right), \text { for } \gamma\left(v, p^{\prime}\right) \leqq p \leqq \frac{p^{\prime}}{p^{\prime}-1} .
$$

The above is a contradiction. An argument similar to the above gives us a contradiction for $p \geqq p^{\prime} / p^{\prime}-1 \geqq \gamma\left(v, p^{\prime}\right)$.

Thus, in order to remove the condition (5.2) one has to somehow improve the bounds in Theorem IV.4.3 and in (4.4.18). Choosing specific configurations in $\overline{\mathscr{E}}_{q, k}$ one may able to show that these bounds are optimal. We first consider the bound in Theorem IV.4.3. To improve the bound one has to improve the bound in Proposition IV.4.1. Consider the following configuration $(\omega)_{n}$ of paths: For a given unit cube $\Delta \subset \Lambda_{q}$, assume that $\omega_{i}\left(\tau_{0}\right) \in \Delta, i=1,2, \ldots, k$, for some $\tau_{0} \in[0, \beta]$. Without loss of generality we may assume $\Delta$ to be the unit cube centered at the origin. 
Then the problem becomes the minimalization of the following expression:

$$
\sum_{r \in A}\left[c_{1} \bar{n}(r, \tau)^{p^{\prime}}+c_{2} \sum_{\omega_{i}(\tau) \in Q(r)} \omega_{i}(\tau)^{2}\right],
$$

which is related to the minimalization of the following non-linear functionals:

$$
H(f) \equiv \int_{d}^{v}(x)\left[f(x)^{p^{\prime}}+f(x) x^{2}\right]
$$

under the restrictions:

$$
f(x) \geqq 0, \quad \int d^{v} x f(x)=K,
$$

where $K$ is a given number. In our case $K=\left|\Lambda_{q}\right|^{l / p}$. Using a variational method the following fact can be proven: The minimum is achieved at

$$
\begin{aligned}
\bar{f}(x) & =\left[\left(c-x^{2}\right) / p^{\prime}\right]^{1 / p^{\prime}-1}, \quad|x| \leqq \sqrt{c}, \\
& =0, \quad|x|>\sqrt{c},
\end{aligned}
$$

where $c$ is determined by (5.4.). From (5.5.) and (5.4) it is now easy to check that there is a constant $b>0$ such that $H(\bar{f})=b K^{\gamma\left(v, p^{\prime}\right)}$. From the above fact we conclude that the bound in Proposition IV.4.1. is optimal.

For (4.4.18) one may choose a specific configuration to show that the bound in (4.4.18) is optimal. Thus, in order to remove the condition (5.2) one may need a further decomposition of $\overline{\mathscr{E}}_{q, k}$ into a union of disjoint subsets.

\section{References}

1. Bratteli, O., Robinson, D. W.: Equilibrium States of a Bose gas with repulsive interactions. Aust J. Math. Series B, to appear

2. Bratteli, O., Robinson, D. W.: Operator algebras and quantum statistical mechanics, I and II, New York: Springer 1981

3. Brydges, D. C., Federbush, P.: The cluster expansion in statistical mechanics. Commun. Math. Phys. 49, 233 (1976); The cluster expansion for potentials with exponential fall-off. 53, 19 (1977)

4. Duneau, M., Souillard, B.: Existence of Green's functions for dilute Bose gases. Commun. Math. Phys. 31, 113 (1973)

5. Fröhlich, J., Park, Y. M.: Correlation inequalities and the thermodynamic limit for classical and quantum continuous systems. II. Bose-Einstein and Fermi-Dirac statistics. J. Stat. Phys. 23, 701 (1980)

6. Ginibre, J.: Some Application of functional integration in statistical mechanics. In: Statistical Mechanics and Field Theory, de Witt, C., Stora R. (eds.). New York: Gordon and Breach 1971

7. Glimm, J., Jaffe, A.: Quantum physics; a functional integral point of view. New York: Springer 1982

8. Lebowitz, J. L., Presutti, E.: Statistical mechanics of systems of unbounded spins. Commun. Math. Phys. 50, 195 (1976)

9. Lieb, E. H., Lebowitz, J. L.: Adv. Math. 9, 316 (1972)

10. Park, Y. M.: Preprint in preparation

11. Reed, M., Simon, B.: Method of modern mathematical physics. Vol. II: Fourier analysis, selfadjointness. New York: Academic Press 1975

12. Ruelle, D. W.: Statistical mechanics. New York, Amsterdam: Benjaminn 1969 
13. Ruelle, D. W.: Superstable interactions in classical statistical mechanics. Commun. Math. Phys. 18, 127 (1970)

14. Ruelle, D. W.: Probability estimates for continuous spin systems. Commun. Math. Phys. 50, 189 (1976)

15. Ruelle, D. W.: Analyticity of Green's functions dilute quantum gases. J. Math. Phys. 12, 901 (1971)

Communicated by J. Fröhlich

Received September 13, 1983 
